Sister Lab Program Prospective Partner Nuclear Profile: Indonesia

Mo Bissani, Sean Tyson

January 12, 2007 
This document was prepared as an account of work sponsored by an agency of the United States Government. Neither the United States Government nor the University of California nor any of their employees, makes any warranty, express or implied, or assumes any legal liability or responsibility for the accuracy, completeness, or usefulness of any information, apparatus, product, or process disclosed, or represents that its use would not infringe privately owned rights. Reference herein to any specific commercial product, process, or service by trade name, trademark, manufacturer, or otherwise, does not necessarily constitute or imply its endorsement, recommendation, or favoring by the United States Government or the University of California. The views and opinions of authors expressed herein do not necessarily state or reflect those of the United States Government or the University of California, and shall not be used for advertising or product endorsement purposes.

This work was performed under the auspices of the U.S. Department of Energy by University of California, Lawrence Livermore National Laboratory under Contract W-7405-Eng-48. 


\section{Sister Lab Program Prospective Partner Nuclear Profile: Indonesia}

December 2006

\section{Background}

Indonesia has participated in cooperative technical programs with the IAEA since 1957, and has cooperated with regional partners in all of the traditional areas where nuclear science is employed: in medicine, public health (such as insect control and eradication programs), agriculture (e.g. development of improved varieties of rice), and the gas and oil industries. Recently, Indonesia has contributed significantly to the Reduced Enrichment Research and Training Reactor (RERTR) Program by conducting experiments to confirm the feasibility of Mo-99 production using high-density low enriched uranium (LEU) fuel, a primary goal of the RERTR Program. ${ }^{1}$

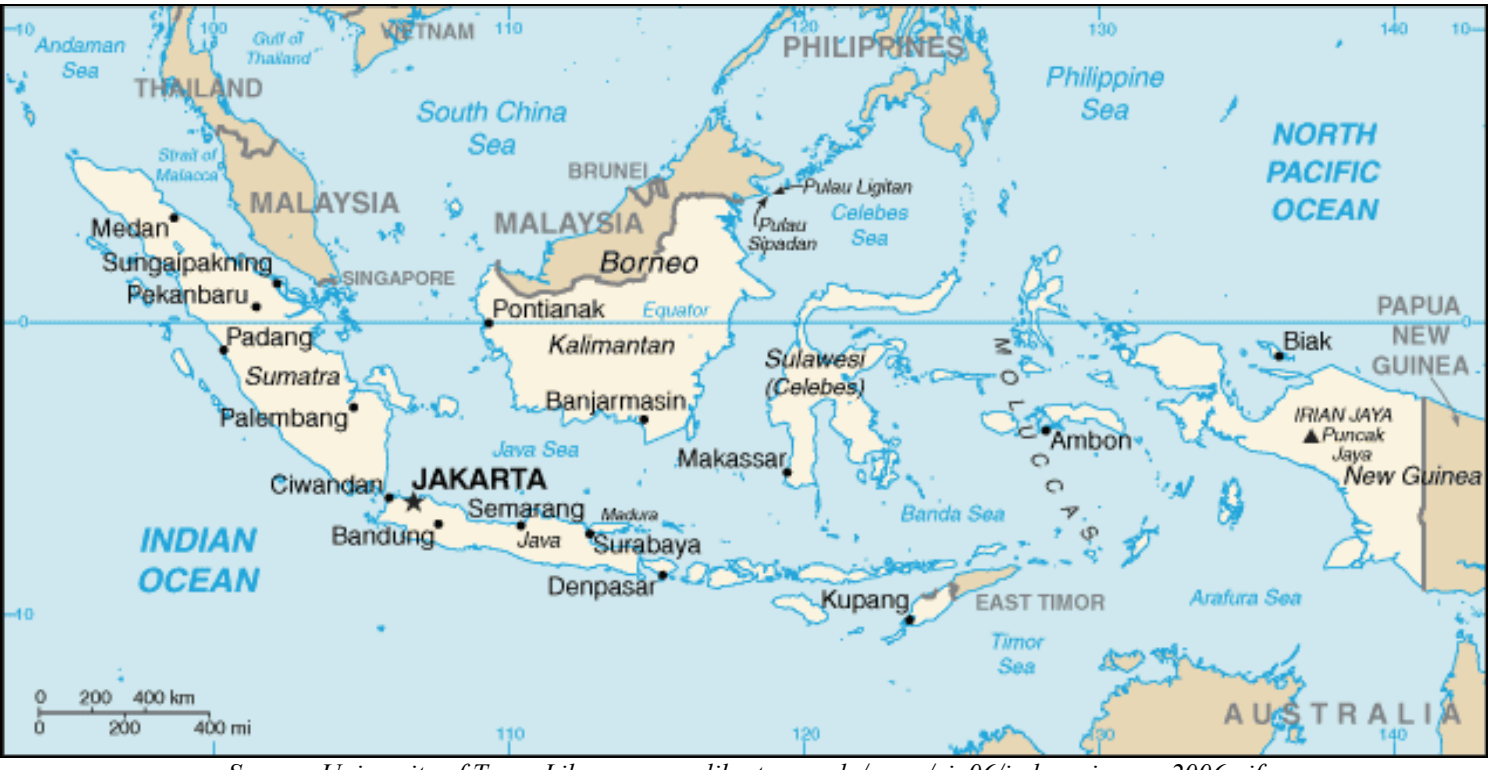

Source: University of Texas Library, www.lib.utexas.edu/maps/cia06/indonesia_sm_2006.gif

Indonesia's first research reactor, the TRIGA Mark II at Bandung, began operation in 1964 at $250 \mathrm{~kW}$ and was subsequently upgraded in 1971 to $1 \mathrm{MW}$ and further upgraded in 2000 to $2 \mathrm{MW}$. This reactor was joined by another TRIGA Mark II, the $100-\mathrm{kW}$ Kartini-PPNY at Yogyakarta, in 1979, and by the 30-MW G.A. Siwabessy multipurpose reactor in Serpong, which achieved criticality in July 1983. A 10-MW radioisotope production reactor, to be called the RPI-10, also was proposed for construction at Serpong in the late $1990 \mathrm{~s},{ }^{2}$ but the project apparently was not carried out.

In the five decades since its nuclear research program began, Indonesia has trained a cadre of scientific and technical staff who not only operate and conduct research with the 
current facilities, but also represent the nucleus of a skilled labor pool to support development of a nuclear power program.

Although Indonesia's previous on-again, off-again consideration of nuclear power has not gotten very far in the past, it now appears that Indonesia again is giving serious consideration to beginning a national nuclear energy program. In June 2006, Research and Technology Minister Kusmayanto Kadiman said that his ministry was currently putting the necessary procedures in place to speed up the project to acquire a nuclear power plant, indicating that, "We will need around five years to complete the project. If we can start the study, go to tender, and sign the contract for the project this year, the power plant could be on stream by 2011 ."3 $^{3}$ While this ambitious schedule may be a bit unrealistic, it suggests new momentum to move forward on the project. The favored site for the proposed plant is the Muria Peninsula, ${ }^{4}$ located on Java's north central coast.

\section{Technical Capabilities and Facilities}

Indonesia has developed small-scale indigenous capabilities in many areas of the nuclear fuel cycle, although it has so far not pursued sensitive areas such as uranium isotope separation or spent-fuel reprocessing. Indonesia has been successful in establishing several significant nuclear facilities, and its research and development activities are supported by an extensive infrastructure of government facilities, research organizations, and university programs.

\section{Uranium Deposits and Yellowcake Production Facilities}

Exploration and Mining

Indonesia has conducted uranium exploration projects, with IAEA assistance, since the early 1980s and has identified uranium deposits thought to be sufficient uranium to meet the yellowcake requirements for its planned nuclear power reactors. ${ }^{5}$ According to the IAEA's World Distribution of Uranium Deposits Database, ${ }^{6}$ Indonesia's known uranium deposits, located in West Kalimantan, currently are dormant.

- The Remaja-Hitam ore body (also known as the Eko-Remaja prospect) is thought to contain $1,000-2,500$ tons of uranium, with a grade of $0.2-0.5$ percent uranium.

- The Rirang-Tanah Merah ore body is believed to contain less than 500 tons of uranium at $1-5$ percent uranium.

- Production of uranium concentrate on a pilot-scale yellowcake using acid leaching and ion exchange was carried out by the Center for Development of Nuclear Ore and Geology at the Lemajung deposit from 1981 to 1986 with a design capacity of 0.2 tU/year. 
Center for Exploration and Processing of Nuclear Materials, Jakarta

With assistance from the IAEA Indonesia's National Nuclear Energy Agency (Badan Tenaga Nuklir Nasional, or BATAN), , in 1985 completed a laboratory for uranium processing at the Center for Exploration and Processing of Nuclear Materials in Jakarta. ${ }^{8}$ One open source reports that "in 1985 this facility received about 175 [sic, no units indicated] of domestically mined uranium ore for processing.",9

\section{Bandung Nuclear Complex}

The Bandung Nuclear Complex, located adjacent to the Bandung Institute of Technology, is Indonesia's oldest nuclear center (established in the early 1960s) and is the site of the first of Indonesia's three research reactors.

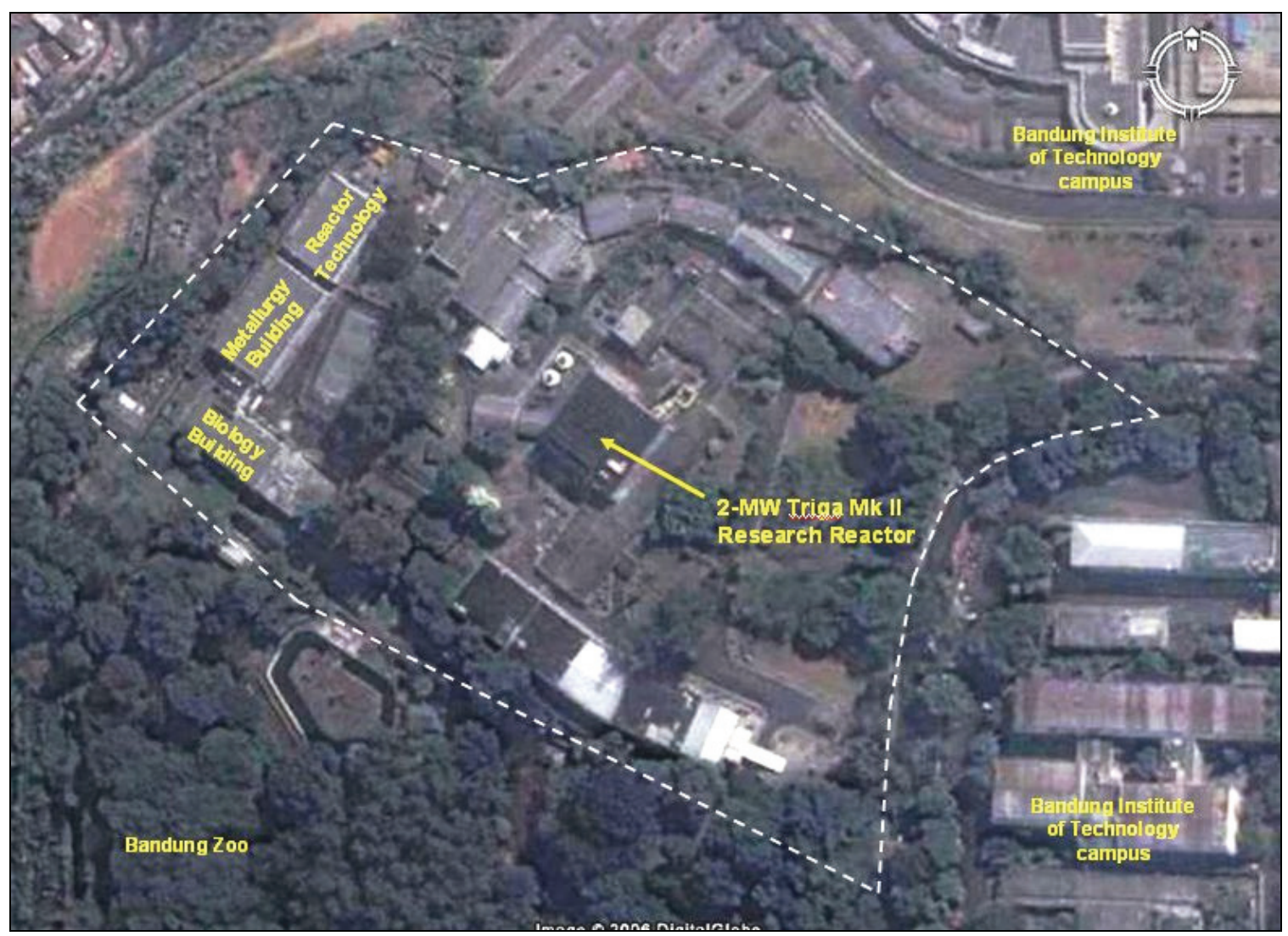

Bandung Nuclear Research Complex, Bandung. Image: DigitalGlobe via Google Earth) Building identifications adapted from: R. Parithusta, "Seismic Hazard Analysis for TRIGA 2000 Nuclear Site, Bandung," $2^{\text {nd }}$ Workshop on Earthquake Engineering for Nuclear Facilities, 2-14 February 2005, International Center for Theoretical Physics, Trieste).

The center has undergone several name changes over the years. Originally called the Bandung Nuclear Research Center (PRAB), the complex became Nuclear Technology Research Center (PPTN) in 1980, and in 1999 became the Center for Research and

\footnotetext{
* In addition to a National Nuclear Energy Agency, Indonesia has a separate national nuclear regulatory authority, BAPETEN, which was established by a 1997 parliamentary act.
} 
Development of Nuclear Technology (P3TkN). Most recently, in a 2006 BATAN reorganization, the name was changed to the Nuclear Technology Center for Materials and Radiometry, or Pusat Teknologi Nuklir Bahan dan Radiometri (PTNBR). The organization chart below is adapted from the PTNBM Web site. ${ }^{10}$

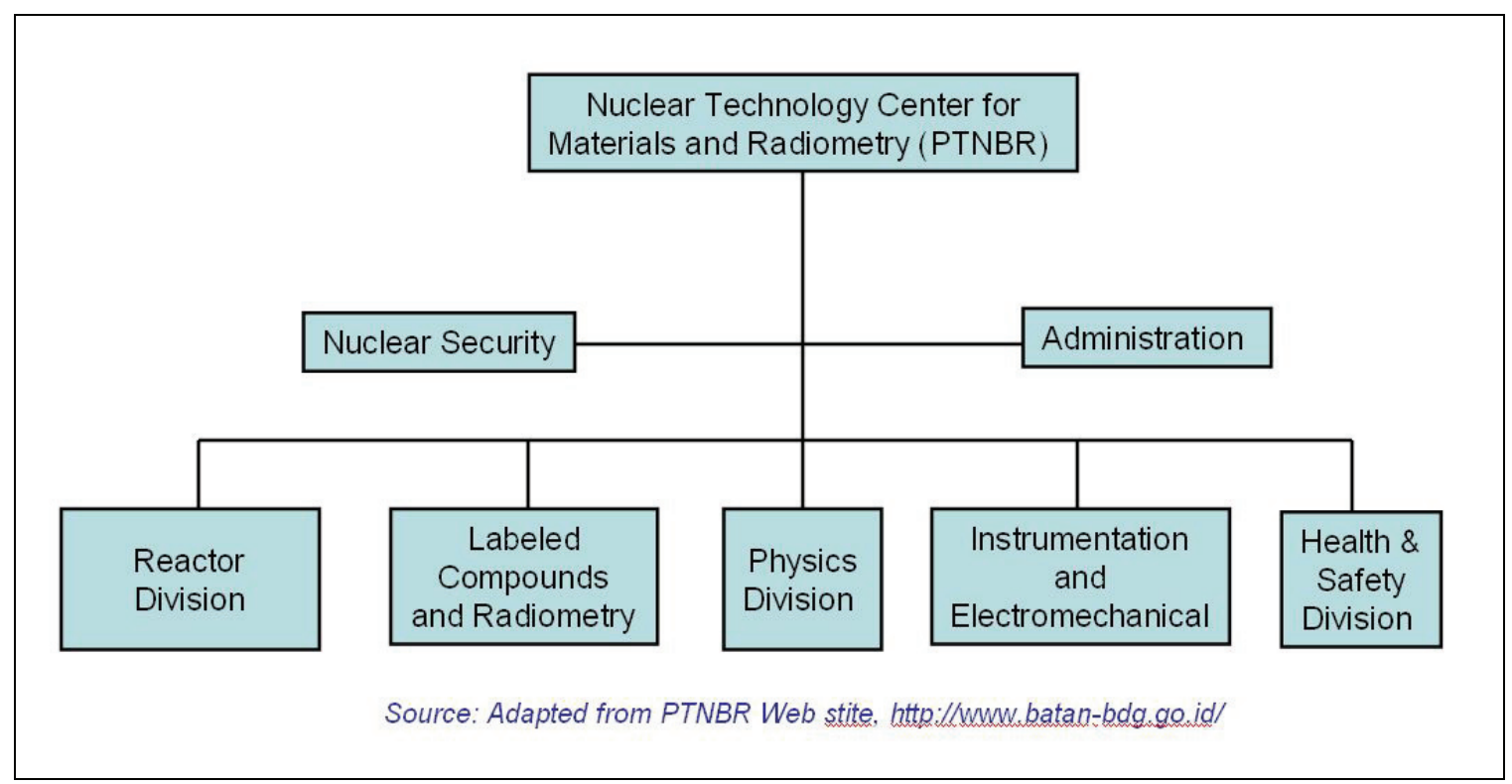

Simplified organization chart for the Bandung nuclear research complex

The Center's Reactor Division operates the 2-MW Bandung TRIGA Mk II research reactor. The reactor achieved criticality 19 October 1964, and has since been used for radioisotope production, irradiation, physics experiments, and training. Its fuel was fabricated by General Atomics of the U.S.

In addition to the reactor and associated facilities, the Bandung center also has conducted activities related to nuclear fuel fabrication. By 1991, with assistance provided under two IAEA Technical Cooperation projects, the center had developed a capability to produce $\mathrm{UO}_{2}$ powder from locally produced yellowcake, fabricate $\mathrm{UO}_{2}$ pellets, and produce short (i.e., not of typical LWR fuel-rod length) fuel pins. ${ }^{11}$ The Center's laboratories also are active in radiochemistry, radiobiology, and radiometallurgy.

\section{Yogyakarta Nuclear Complex}

BATAN's nuclear research complex at Yogyakarta is currently called the Center for Accelerator Technology and Material Processes, or PTAPB for its Indonesian name, Pusat Teknologi Akselerator dan Proses Bahan. . The Center's Reactor Division operates the 100-kW Kartini TRIGA Mark II research reactor and an associated critical assembly. Other facilities include a laboratory for pure materials research, various accelerators, and laboratories for nuclear physics and chemistry. The Center's research activities include

\footnotetext{
* The Center formerly was called the Center of Research and Development for Advanced Technology, or Pusat Penelitian dan Pengembangan Teknologi Maju (P3TM).
} 
R\&D in nuclear physics, chemistry, low- and medium-energy particle accelerator technology, process technology, analysis of nuclear materials, the use of the reactor for research and training. ${ }^{12}$

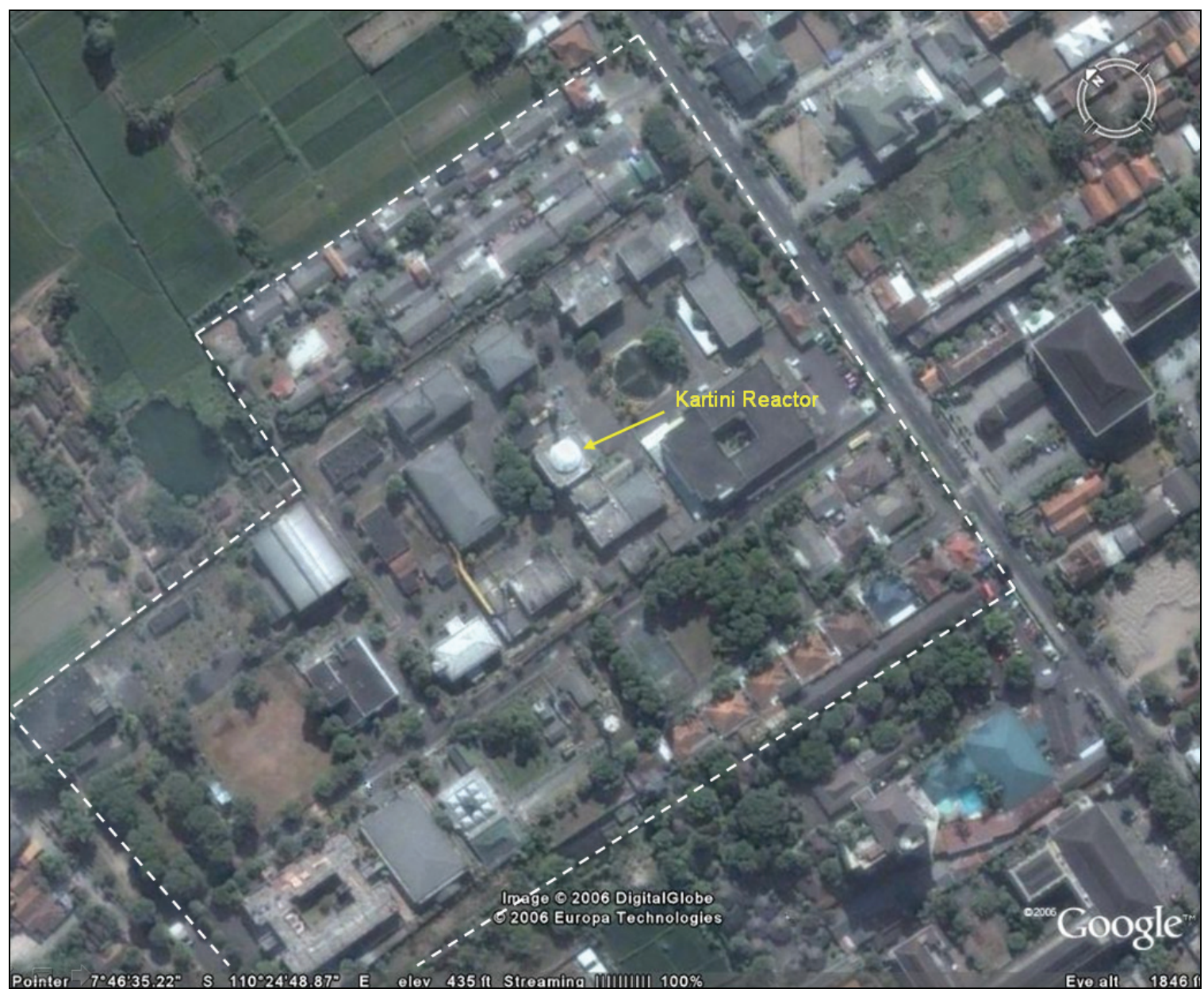

Center for Accelerator Technology and Material Processes (PTAPB), Yogyakarta, site of the Kartini research reactor. (Image: DigitalGlobe via Google Earth)

\section{Serpong Nuclear Complex}

The Serpong nuclear site is a diverse research complex for nuclear technology development. It contains centers for research, development, and engineering of nuclear science and technology. It was built from 1983 -1992 the objectives of supporting development of Indonesian nuclear industry and preparing for development and operation of nuclear power plants in Indonesia. The Serpong nuclear complex is located $25 \mathrm{~km}$ southwest of Jakarta and $3 \mathrm{~km}$ south of the town of Serpong, within the larger National Center for Research Science and Technology (PUSPIPTEK) complex. The main nuclear facility at the site is the 30-MW "G.A. Siwabessy" multipurpose reactor. Other significant installations include a laboratory for fabrication of power reactor fuel, a production facility for research reactor fuel elements, a Development Center for 
Radioactive Waste Management, as well as facilities for radiometallurgy, reactor safety, neutron spectrometry, nuclear information, and computer technology. ${ }^{13}$

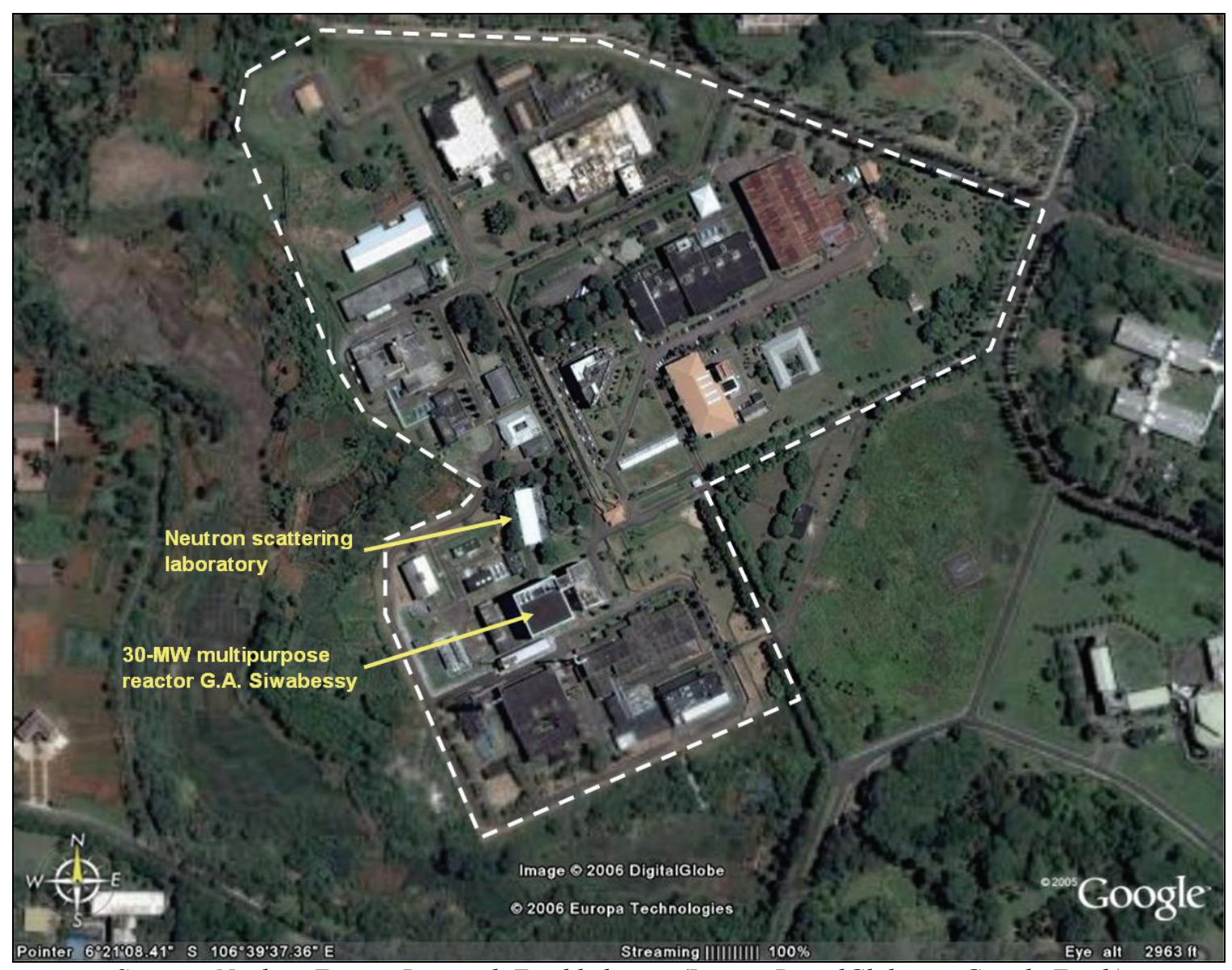

Serpong Nuclear Energy Research Establishment (Image: DigialGlobe via Google Earth)

The 30-MW G.A. Siwabessy reactor is a German-built, pool-type materials test reactor, which first achieved criticality 29 July 1987, and has since been used for basic research, radioisotope and radiopharmaceutical production, material science, silicon doping, nuclear engineering activities, and education in reactor operation and maintenance. Its U3O8 fuel initially was supplied by Germany but now is fabricated at Serpong using imported 20\% uranium. Exploratory efforts are underway to fabricate silicide fuel for the reactor.

In addition to research reactor fuel fabrication, the Serpong site also has a laboratory for fabrication of $\mathrm{UO}_{2}$ fuel pellets and pins.

Radioactive waste treatment, storage, and disposal studies are all conducted at the nuclear Development Center for Radioactive Waste Management. In 1990, Indonesia, in collaboration with the IAEA, completed a six year project to improve its radioactive waste management program for the disposal of radioactive waste from its research reactors. Indonesia is currently thought to operate in accordance with international norms. 


\section{Pasar Jum'at Nuclear Center}

The Center for the Application of Isotope and Radiation Technologies, or Pusat Aplikasi Teknologi Isotop dan Radiasi (PATIR), is located in the Pasar Jum'at area of southern Jakarta. It was established in 1966. The site includes no nuclear research reactor, but there are several cobalt-60 gamma irradiators $(90 \mathrm{kCi}, .60 \mathrm{kCi}, 7.5 \mathrm{kCi}$, and $0.3 \mathrm{kCi})$ as well as electron beam sources for irradiation and isotope production technology. The center has a laboratory for nuclear safety and nuclear biomedicine, and provides nuclearrelated education and training. ${ }^{14}$

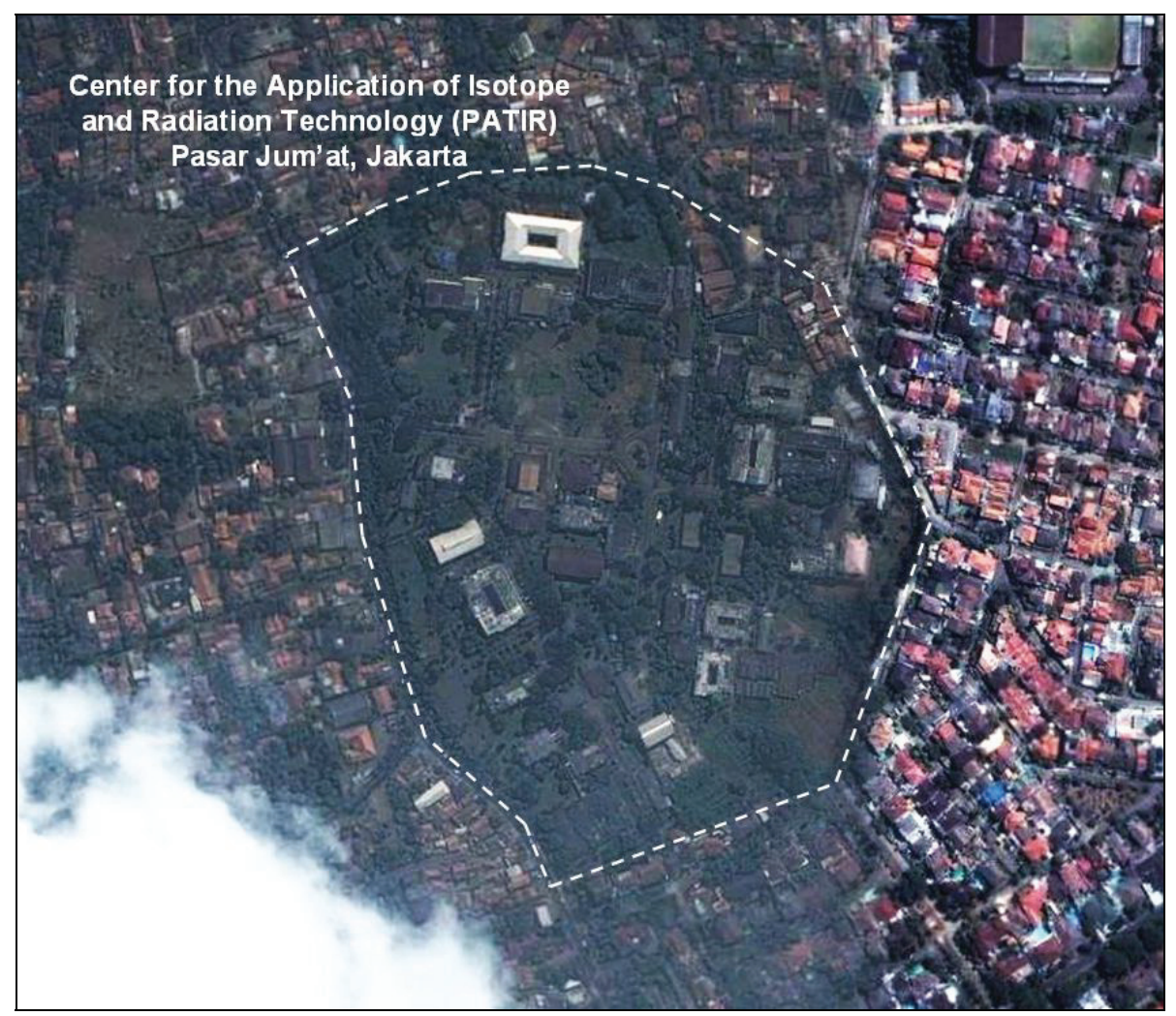

Pasar Jum'at nuclear research center (Image: DigialGlobe via Google Earth)

\section{Nuclear Power}

A rapid increase in domestic energy demand, coupled with the limited sustainability of current resources, has led to a need for new sources of energy. While gas and oil will continue to be the main sources, the commissioning of nuclear power plants would help stabilize the supply of electricity and conserve oil and coal reserves.

A study on national energy planning was conducted in 2001. The study's objective was to support the planning and decision-making process in Indonesia's energy and electricity

\footnotetext{
* Formerly called the Pasar Jumat Center for Nuclear Technology Research (PPTN-Pasar Jumat)
} 
sectors, taking into account economic, environmental, and social aspects. The study team comprised members from the following Indonesian institutions: the Agency of Assessment and Application of Technology (BPPT), the National Nuclear Energy Agency (BATAN), the Directorate General of Electricity and Energy Development (DJLPE), the Directorate General of Oil and Gas (DJMIGAS), the Environmental Impact Control Agency (BAPEDAL), the National Center for Statistics (BPS), the State Electricity Company (PT. PLN Persero), and the Non-Governmental Organization for Environment (NGO). The International Atomic Energy Agency (IAEA) assisted the Government of Indonesia in executing this study by giving aid in the form of computer software and assistance from its experts.

The results of this study provided a projection for energy demand taking into consideration population growth, economic growth, and technology. The Gas Base-load Plant was ranked first in technology for electricity generation. The Coal Base-load Plant came in second due to economically competitive electricity generation. Introduction of nuclear power will become more favorable as the use of fossil fuels is constrained and environmental standards are enforced; thus, nuclear power was ranked third in the study. The potential for growth in natural gas exports is promising, particularly if nuclear power is introduced in Java-Bali, allowing for a reduction in domestic consumption.

BAPETEN has prepared a roadmap for introducing the construction of nuclear power plants (Figure 2). The roadmap is derived from the National Energy Policy of 2004, and includes the following objectives:

- Assess the capability of the electrical grid to accommodate the electricity produced by NPPs.

- Reach a nuclear energy capacity of 6000-7000 MWe by 2025, meeting 6-7\% of estimated total national electricity demand.

- Ensure that Act No. 20 (2002) concerning electricity is consistently applied.

Current plans estimate a first nuclear power reactor will be built as early as 2011 (a somewhat unrealistically ambitious date) followed by at least four more by $2025 .{ }^{15}$ The vendor for the reactors has not been settled on, with offers being entertained from France, South Korea and Japan. The Indonesian government also plans to construct nuclear plants in remote and more rural areas, and to this end Russia has proposed floating nuclear reactors. Indonesia has expressed interest, but there has been little indication that it intends to move forward with this option. ${ }^{16}$

\section{Nuclear Institutions}

\section{Nuclear Energy Control Board (NECB, or BAPETEN)}

BAPETEN was established on 8 May 1998 by Presidential Decree No. 76, and began operations 1 January 1999. ${ }^{17}$ The Nuclear Energy Act of 1997 replaced Act No. 31 of 1964, declaring a requirement for the government to establish regulations, issue licenses, perform inspections, and undertake necessary enforcement in order to ensure the compliance of nuclear energy operators with safety regulations and requirements. 
BAPETEN carries out these functions as an independent government agency that is responsible directly to the President of Indonesia.

\section{National Nuclear Energy Agency (NNEA, or BATAN) ${ }^{18}$}

BATAN was also established by Presidential Decree No. 76. According to its website, BATAN is an independent government agency charged with supporting nuclear research and development that will contribute to the development of nuclear energy in Indonesia. BATAN supports training and maintaining a pool of experts in nuclear science and technology, and operating nuclear facilities that benefit agriculture, industry, and the scientific community. ${ }^{19}$

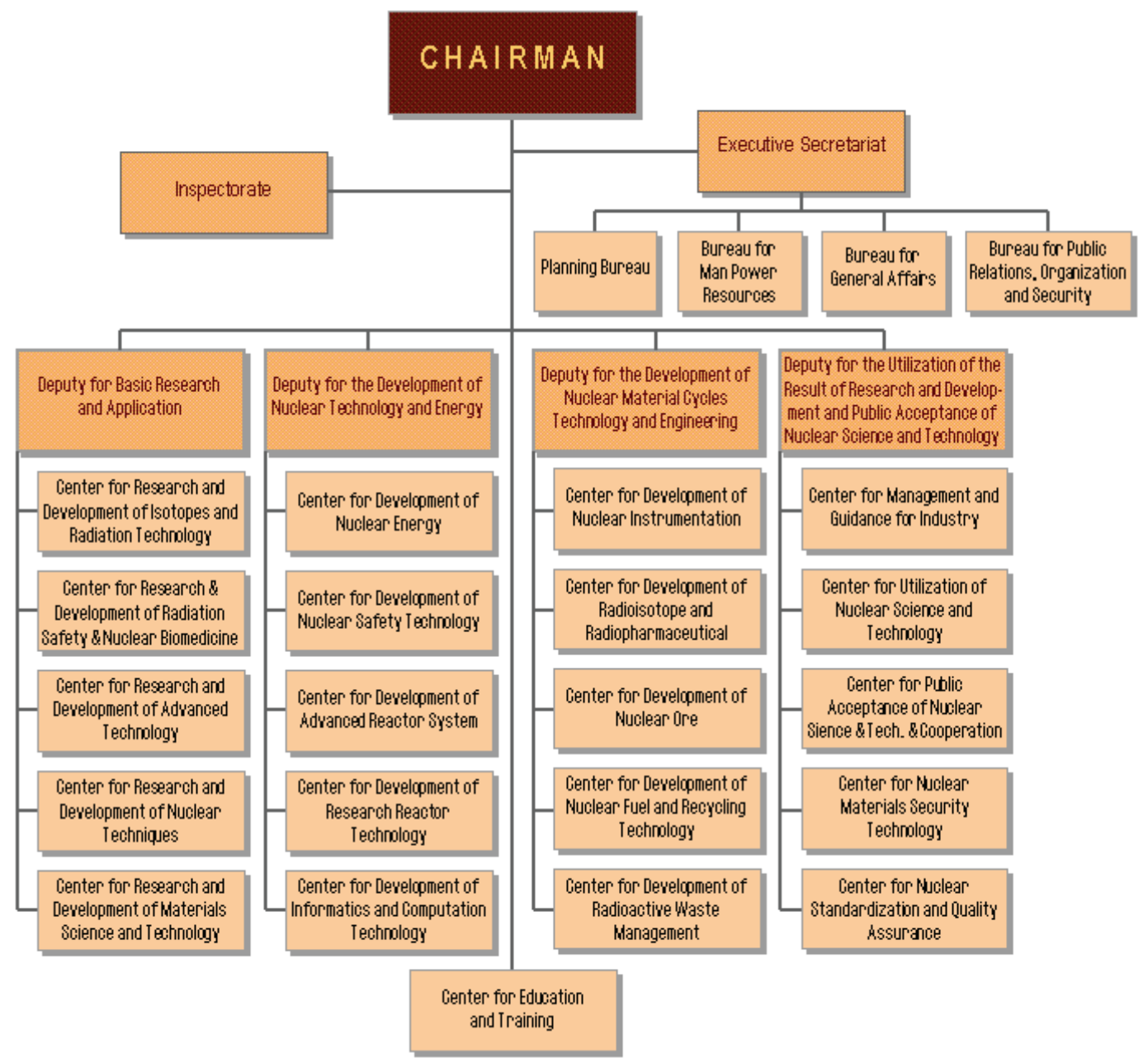

Source: BATAN, www.batan.go.id/batan.html 
Other Institutions:

Other government institutions involved in nuclear policy and activities include the following, listed on the IAEA directory of Indonesia Nuclear Authorities. ${ }^{20}$

Department of Mines and Energy (Jakarta)

The Department of Mines and Energy has regulatory authority over the uranium mines.

Ministry of State for Research and Technology

The State Ministry of Research and Technology was established in 1962. It has the responsibility to assist the President of the Republic of Indonesia in formulating national policies and implementing coordination in the field of research, science and technology. BATAN and BAPETAN are both non-departmental agencies that function beneath the Ministry, as do the Center for Research, Science and Technology (PUSPIPTEK) and the Indonesian Institute of Sciences (LIPI). ${ }^{21}$ 


\section{Bilateral and Multilateral Nuclear Technical Cooperation}

Indonesia has a long history of technical cooperation with the IAEA and with other countries, both regional and around the globe. Its nuclear research program is well established, and spans nearly five decades. With four nuclear research facilities in operation (including three operational research reactors), a cadre of trained professionals and ties to the IAEA, foreign capitals, universities, and research labs, the country is able to offer a great deal of expertise across almost an entire spectrum to other states exploring nuclear technology.

Indonesia has sponsored the Asian Nuclear Safety Network (ANSN), a computer network jointly operated by BAPETEN and BATAN. The objective of the ANSN project is to pool and share existing and new technical knowledge and practical experience to further improve the safety of nuclear installations in Asia. The ANSN computer network is operated through eight ANSN National Centers in China, Indonesia, Japan, Korea, Malaysia, the Philippines, Thailand and Vietnam. Each National Center has a web site associated with it, which can provide access to information regarding nuclear safety and serves as a portal to other ANSN sites. ANSN can be searched locally or through the IAEA's web site. ${ }^{22}$

In addition to projects conducted through the IAEA, Indonesian officials and technical staff have also recently been involved with the U.S. Department of Energy's International Radiological Threat Reduction (IRTR) Program. ${ }^{23}$ At four nuclear sites, U.S. and Indonesian personnel implemented upgrades to the physical security and material accounting systems in place. All work was completed by FY 05, and projects associated with the four sites entered sustainability mode in FY $06 .^{24}$

\subsection{National Projects:}

\begin{tabular}{|c|c|c|c|c|c|c|}
\hline $\begin{array}{c}\text { Active Na } \\
\text { Project } \\
\text { Number }\end{array}$ & $\begin{array}{l}\text { Projects (12) } \\
\text { Title }\end{array}$ & Field & $\begin{array}{l}\text { 1st Year of } \\
\text { Approval }\end{array}$ & Country Officer & $\begin{array}{l}\text { Project Responsible } \\
\text { Officer }\end{array}$ & Technical Officer \\
\hline INS5030 & Sustainable Agricultural Development in Yogyakarta & $5 \mathrm{~A}$ & 2001 & $\begin{array}{l}\text { Sukasam, Kesrat } \\
\text { (TCAP) }\end{array}$ & $\begin{array}{l}\text { Sukasam, Kesrat } \\
\text { (TCAP) }\end{array}$ & $\begin{array}{l}\text { Spencer, Marie } \\
\text { Madeleine (NAFA) } \\
\text { Lagoda, Pierre Jean } \\
\text { Laurent (NAFA) }\end{array}$ \\
\hline
\end{tabular}

Total Budget

158510 
Improving Beef and Dairy Cattle Production in Yogyakarta

Groundwater Resources Exploitation in the Gunung Kidul Area

INS8023

INS0017

INS6011

INS6012

Ruman Resource Development and Nuclear

Technology Support

Detecting Drug-resistant Strains of Tuberculosis

Initiation of Radiotherapy Center in Kalimantan

INS9022

Inspection Procedures and Methods for Assessing

Reactor Tank Liners

INS4033

Preparation for a Nuclear Power Plant

INS4034

INS4035

Feasibility Study for Nuclear Desalination Plant Construction

Preparation of Regulations, Codes, Guides and Standards for a Nuclear Power Plant

INS7004
Utilization of Neutron Generator Accelerator for Environmental Monitoring
2001

Sukasam, Kesrat (TCAP)

$8 \mathrm{M}$

\section{Sukasam, Kesrat (TCAP)}

2003 Sukasam, Kesrat (TCAP)

2003 Sukasam, Kesrat (TCAP)

2003 Sukasam, Kesrat (TCAP) (TCAP) (TCAP) (TCAP)
2001 Sukasam, Kesrat (TCAP)
2003 Sukasam, Kesrat (TCAP)

2005 Sukasam, Kesrat

2005 Sukasam, Kesrat

2005 Sukasam, Kesrat

2005 Sukasam, Kesrat (TCAP)
Sukasam, Kesrat (TCAP)

Spencer, Marie

Madeleine (NAFA)

Miranda, Manoela

Pessoa de (NAFA)

Lagoda, Pierre Jean

Laurent (NAFA)

Sukasam, Kesrat (TCAP)

Sukasam, Kesrat (TCAP)

Sukasam, Kesrat (TCAP)

Sukasam, Kesrat (TCAP)

Sukasam, Kesrat (TCAP)

Sukasam, Kesrat (TCAP)

Sukasam, Kesrat (TCAP)

Sukasam, Kesrat (TCAP)

Sukasam, Kesrat (TCAP)

Sukasam, Kesrat (TCAP)
Boettcher, Paul John

(NAFA)

Vercoe, Philip Edward (NAFA)

Ito, Mari (NAPC)

Dias, Mahendra

Prinath (TCAP)

Khan, Baldip Kaur

(NAHU)

Rosenblatt, Eduardo

(NAHU)

McLean, Ian Donald (NAHU)

Paranjpe, Shriniwas

Krishnarao (NAPC)

Ciuculescu, Cristina

(NSNI)

Cheng, Huiping

(NENP)

Misra, Braj Mohan

(NENP)

Gasparini, Marco

(NSNI)

Dytlewski, Nikolai

NAPC)

Rossbach, Matthias $\mathrm{H}$.

(NAPC)
180940

120671

75627

463374

267210

699456

457920

116980

33790

77280

147990 


\begin{tabular}{|c|c|}
\hline \multicolumn{2}{|c|}{ Completed National Projects (123) } \\
\hline $\begin{array}{l}\text { Project } \\
\text { Number }\end{array}$ & Title \\
\hline INS9005 & Environmental Radioactivity \\
\hline INS4007 & Reactor Engineering \\
\hline INS3003 & Raw Materials Prospection \\
\hline INS5011 & Radioisotopes in Animal Science \\
\hline INS4011 & Radiation Measurements Instrumentation \\
\hline INS3004 & Raw Materials Prospection \\
\hline INS5014 & Radioisotopes in Agriculture \\
\hline INS4009 & Nuclear Metallurgy \\
\hline INS5013 & Mutation Breeding \\
\hline INS1006 & Materials Research \\
\hline INS2007 & Radiochemistry \\
\hline INS4014 & Reactor Engineering \\
\hline INS4016 & Reactor Electronics \\
\hline INS1007 & Nuclear Magnetic Resonance \\
\hline INS2006 & X-Ray Fluorescence \\
\hline
\end{tabular}

\begin{tabular}{|c|c|c|}
\hline Field & $\begin{array}{l}\text { Completed } \\
\text { on }\end{array}$ & Country Officer \\
\hline $\begin{array}{l}9 \mathrm{Q} \\
9 \mathrm{C}\end{array}$ & $1976-10-01$ & $\begin{array}{l}\text { Nataadidjaja, Erman } \\
\text { (TC) }\end{array}$ \\
\hline $4 \mathrm{E}$ & $1977-10-12$ & $\begin{array}{l}\text { Nataadidjaja, Erman } \\
\text { (TC) }\end{array}$ \\
\hline $3 B$ & $1977-12-22$ & $\begin{array}{l}\text { Nataadidjaja, Erman } \\
\text { (TC) }\end{array}$ \\
\hline $5 \mathrm{E}$ & $1978-03-28$ & $\begin{array}{l}\text { Nataadidjaja, Erman } \\
\text { (TC) }\end{array}$ \\
\hline $4 G$ & 1978-03-29 & $\begin{array}{l}\text { Nataadidjaja, Erman } \\
\text { (TC) }\end{array}$ \\
\hline $3 B$ & $1978-10-13$ & $\begin{array}{l}\text { Nataadidjaja, Erman } \\
\text { (TC) }\end{array}$ \\
\hline $5 B$ & $1979-10-10$ & $\begin{array}{l}\text { Nataadidjaja, Erman } \\
\text { (TC) }\end{array}$ \\
\hline $4 \mathrm{E}$ & $1980-02-22$ & $\begin{array}{l}\text { Nataadidjaja, Erman } \\
\text { (TC) }\end{array}$ \\
\hline $5 C$ & $1980-02-22$ & $\begin{array}{l}\text { Nataadidjaja, Erman } \\
\text { (TC) }\end{array}$ \\
\hline $1 \mathrm{E}$ & $1980-05-20$ & $\begin{array}{l}\text { Nataadidjaja, Erman } \\
\text { (TC) }\end{array}$ \\
\hline $2 \mathrm{~B}$ & $1980-05-20$ & $\begin{array}{l}\text { Nataadidjaja, Erman } \\
\text { (TC) }\end{array}$ \\
\hline $4 \mathrm{D}$ & $1980-05-20$ & $\begin{array}{l}\text { Nataadidjaja, Erman } \\
\text { (TC) }\end{array}$ \\
\hline $4 G$ & $1980-05-23$ & $\begin{array}{l}\text { Nataadidjaja, Erman } \\
\text { (TC) }\end{array}$ \\
\hline $1 G$ & $1980-07-14$ & $\begin{array}{l}\text { Nataadidjaja, Erman } \\
\text { (TC) }\end{array}$ \\
\hline $2 \mathrm{C}$ & $1980-08-26$ & $\begin{array}{l}\text { Nataadidjaja, Erman } \\
\text { (TC) }\end{array}$ \\
\hline
\end{tabular}

\section{Project Responsible Technical Officer} Officer

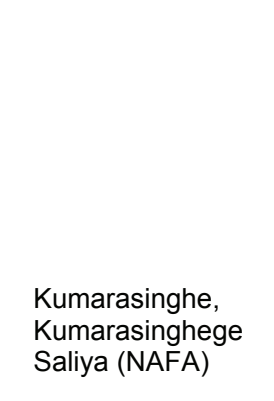

a

\section{Total Budget}

18802

31214

66344

30237

64544

82631

14809

8562

36530

47194

24113

6905

16116

35960

46918 


\begin{tabular}{|c|c|c|c|c|}
\hline INS4013 & Irradiation Studies Using King Furnace & $4 \mathrm{~K}$ & $1980-08-26$ & $\begin{array}{l}\text { Nataadidjaja, Erman } \\
\text { (TC) }\end{array}$ \\
\hline INS5015 & Fish Irradiation Preservation & $5 \mathrm{H}$ & $1980-09-25$ & $\begin{array}{l}\text { Nataadidjaja, Erman } \\
\text { (TC) }\end{array}$ \\
\hline INS5016 & Radioisotopes in Animal Science & $5 \mathrm{E}$ & $1980-09-30$ & $\begin{array}{l}\text { Nataadidjaja, Erman } \\
\text { (TC) }\end{array}$ \\
\hline INS2008 & Labelled Compounds & $2 \mathrm{~F}$ & $1981-09-08$ & $\begin{array}{l}\text { Nataadidjaja, Erman } \\
\text { (TC) }\end{array}$ \\
\hline INS2009 & Labelled Compounds & $2 \mathrm{~F}$ & $1981-09-08$ & $\begin{array}{l}\text { Nataadidjaja, Erman } \\
\text { (TC) }\end{array}$ \\
\hline INS4015 & Nuclear Fuel Fabrication & $4 \mathrm{E}$ & $1981-09-08$ & $\begin{array}{l}\text { Nataadidjaja, Erman } \\
\text { (TC) }\end{array}$ \\
\hline INS8010 & Radiation Processing & $8 \mathrm{H}$ & $1981-09-09$ & $\begin{array}{l}\text { Nataadidjaja, Erman } \\
\text { (TC) }\end{array}$ \\
\hline INS8008 & Consultancies on Radiation Processing for Industry & $\begin{array}{l}8 \mathrm{H} \\
8 \mathrm{I}\end{array}$ & $1982-06-30$ & $\begin{array}{l}\text { Nethsinghe, Douglas } \\
\text { A. (TCPM) }\end{array}$ \\
\hline INS1011 & Ion Implantation Technique & $1 G$ & $1982-07-20$ & $\begin{array}{l}\text { Nataadidjaja, Erman } \\
\text { (TC) }\end{array}$ \\
\hline INS3006 & Uranium Exploration & $3 C$ & $1982-07-20$ & $\begin{array}{l}\text { Nataadidjaja, Erman } \\
\text { (TC) }\end{array}$ \\
\hline INS4012 & Nuclear Electronics & $4 G$ & $1982-07-20$ & $\begin{array}{l}\text { Nataadidjaja, Erman } \\
\text { (TC) }\end{array}$ \\
\hline INS1008 & Laser Instrumentation & $1 C$ & $1983-09-23$ & $\begin{array}{l}\text { Nataadidjaja, Erman } \\
\text { (TC) }\end{array}$ \\
\hline INS8009 & Isotopes in Hydrology & $8 \mathrm{~N}$ & $1983-09-23$ & $\begin{array}{l}\text { Nataadidjaja, Erman } \\
\text { (TC) }\end{array}$ \\
\hline INS5017 & Radioisotopes in Poultry Nutrition & $5 \mathrm{E}$ & $1983-11-21$ & $\begin{array}{l}\text { Nataadidjaja, Erman } \\
\text { (TC) }\end{array}$ \\
\hline
\end{tabular}

Colton, John $\mathrm{P}$

(TCPC)

Loaharanu, Paisan

(NAFA)

Young, Bruce A

(NAFA)

Dargie, James Donald

(NAFA)

Vera Ruiz, Hernan

Guido (NAPC)

Yuan, Hong-Chien

(TCAC)

Colton, John P.

(TCPC)

Sukhanov, Georgy

(NENP)

Yuan, Hong-Chien

(TCAC)

Machi, Sueo (NA)

Yuan, Hong-Chien

(TCAC)

Dolnicar, Joze (NAPC)

Tauchid, Mohamad

(NEFW)

Barretto de Campos,

Paulo Marcos (TCPB)

Dolnicar, Joze (NAPC)

Vuister, Petrus

Hendrikus (RILS)

Dolnicar, Joze (NAPC)

Payne, Bryan (NAPC)

Dargie, James Donald (NAFA)
15558

17203

64766

12605

49453

7432

15372

12627

9018

34897

27234

27559

3781

49151 


\begin{tabular}{|c|c|}
\hline INS4019 & Nuclear Electronics \\
\hline INS1009 & Secondary Standards Dosimetry Laboratory \\
\hline INS6003 & Radioimmunoassay Services \\
\hline INS1013 & Nuclear Magnetic Resonance (NMR) \\
\hline INS3007 & Uranium Ore Processing \\
\hline INS1012 & Neutron Scattering Study \\
\hline INS2010 & Production of Radioimmunoassay Kits \\
\hline INS8012 & Neutron Radiography \\
\hline INS0006 & $\begin{array}{l}\text { Reorganization of Batan's Management and } \\
\text { Administration }\end{array}$ \\
\hline INS0005 & Nuclear Manpower Development \\
\hline INS3005 & Nuclear Materials Analysis \\
\hline INS4020 & Nuclear Instrument Maintenance \\
\hline INS1010 & Secondary Standards Dosimetry Laboratory \\
\hline INS9009 & Radiation Physics \\
\hline
\end{tabular}

\begin{tabular}{|c|c|c|}
\hline $4 G$ & $1985-06-03$ & $\begin{array}{l}\text { Nataadidjaja, Erman } \\
\text { (TC) }\end{array}$ \\
\hline $1 \mathrm{~K}$ & $1985-06-25$ & $\begin{array}{l}\text { Nataadidjaja, Erman } \\
\text { (TC) }\end{array}$ \\
\hline $6 B$ & $1985-06-25$ & $\begin{array}{l}\text { Nataadidjaja, Erman } \\
\text { (TC) }\end{array}$ \\
\hline $1 G$ & $1985-08-26$ & $\begin{array}{l}\text { Nataadidjaja, Erman } \\
\text { (TC) }\end{array}$ \\
\hline $3 F$ & $1985-10-21$ & $\begin{array}{l}\text { Islam, Mohammad } \\
\text { Mizanul (TCPA) }\end{array}$ \\
\hline $1 \mathrm{E}$ & $1986-06-27$ & $\begin{array}{l}\text { Islam, Mohammad } \\
\text { Mizanul (TCPA) }\end{array}$ \\
\hline $\begin{array}{l}2 \mathrm{G} \\
2 \mathrm{~F}\end{array}$ & $1986-06-27$ & $\begin{array}{l}\text { Islam, Mohammad } \\
\text { Mizanul (TCPA) }\end{array}$ \\
\hline $\begin{array}{l}8 \mathrm{C} \\
1 \mathrm{~L} \\
4 \mathrm{E}\end{array}$ & $1986-07-25$ & $\begin{array}{l}\text { Islam, Mohammad } \\
\text { Mizanul (TCPA) }\end{array}$ \\
\hline $0 G$ & $1987-03-16$ & $\begin{array}{l}\text { Nethsinghe, Douglas } \\
\text { A. (TCPM) }\end{array}$ \\
\hline 이 & $1987-10-30$ & $\begin{array}{l}\text { Islam, Mohammad } \\
\text { Mizanul (TCPA) }\end{array}$ \\
\hline $3 E$ & $1987-10-30$ & $\begin{array}{l}\text { Islam, Mohammad } \\
\text { Mizanul (TCPA) }\end{array}$ \\
\hline $4 G$ & $1987-10-30$ & $\begin{array}{l}\text { Islam, Mohammad } \\
\text { Mizanul (TCPA) }\end{array}$ \\
\hline $1 \mathrm{~K}$ & $1988-12-19$ & $\begin{array}{l}\text { Islam, Mohammad } \\
\text { Mizanul (TCPA) }\end{array}$ \\
\hline $\begin{array}{l}6 \mathrm{~F} \\
9 \mathrm{C}\end{array}$ & $1988-12-19$ & $\begin{array}{l}\text { Islam, Mohammad } \\
\text { Mizanul (TCPA) }\end{array}$ \\
\hline
\end{tabular}

Vuister, Petrus

Hendrikus (RILS)

Haider, Johann Georg

(TCPM)

Dudley, Robert A.

(RILS)

Dolnicar, Joze (NAPC)

Ajuria Garza, Sergio (NENF)

Tauchid, Mohamad

(NEFW)

Dolnicar, Joze (NAPC)

Piyasena, Rienzil

Dodwell (NAHU)

Muranaka, Richard

George G. (NAPC)

Nethsinghe, Douglas

A. (TCPM)

L'Annunziata, Michael

Frank (TC)

Noe, Maxy Charles

(NENF)

Vuister, Petrus

Hendrikus (RILS)

Haider, Johann Georg (TCPM)

Van Herk, Gerard (NAHU)
28331

37757

34538

63653

32067

155868

54129

10214

4602

22251

57483

63607

326220

8419 
INS0004_Nuclear Materials Accounting

INS1017 Nuclear Analytical Techniques

INS4018 Reactor Physics

\begin{tabular}{|c|c|c|}
\hline OB & 1989-06-15 & $\begin{array}{l}\text { Islam, Mohammad } \\
\text { Mizanul (TCPA) }\end{array}$ \\
\hline $\begin{array}{l}8 \mathrm{H} \\
8 \mathrm{I}\end{array}$ & $1989-09-25$ & $\begin{array}{l}\text { Islam, Mohammad } \\
\text { Mizanul (TCPA) }\end{array}$ \\
\hline $8 \mathrm{M}$ & $1989-09-29$ & $\begin{array}{l}\text { Islam, Mohammad } \\
\text { Mizanul (TCPA) }\end{array}$ \\
\hline $0 \mathrm{~J}$ & $1989-10-30$ & $\begin{array}{l}\text { Islam, Mohammad } \\
\text { Mizanul (TCPA) }\end{array}$ \\
\hline $1 G$ & $1989-10-30$ & $\begin{array}{l}\text { Islam, Mohammad } \\
\text { Mizanul (TCPA) }\end{array}$ \\
\hline $6 B$ & $1989-10-30$ & $\begin{array}{l}\text { Islam, Mohammad } \\
\text { Mizanul (TCPA) }\end{array}$ \\
\hline $9 G$ & $1989-10-30$ & $\begin{array}{l}\text { Islam, Mohammad } \\
\text { Mizanul (TCPA) }\end{array}$ \\
\hline $0 \mathrm{C}$ & $1990-12-20$ & $\begin{array}{l}\text { Islam, Mohammad } \\
\text { Mizanul (TCPA) }\end{array}$ \\
\hline $\begin{array}{r}1 \mathrm{~L} \\
4 \mathrm{~A}\end{array}$ & $1990-12-20$ & $\begin{array}{l}\text { Islam, Mohammad } \\
\text { Mizanul (TCPA) }\end{array}$ \\
\hline $\begin{array}{l}4 \mathrm{D} \\
1 \mathrm{~F}\end{array}$ & $1990-12-20$ & $\begin{array}{l}\text { Islam, Mohammad } \\
\text { Mizanul (TCPA) }\end{array}$ \\
\hline $\begin{array}{l}4 \mathrm{~B} \\
1 \mathrm{~F}\end{array}$ & $1990-12-20$ & $\begin{array}{l}\text { Islam, Mohammad } \\
\text { Mizanul (TCPA) }\end{array}$ \\
\hline 3B & $1990-12-22$ & $\begin{array}{l}\text { Islam, Mohammad } \\
\text { Mizanul (TCPA) }\end{array}$ \\
\hline $8 \mathrm{C}$ & $1990-12-22$ & $\begin{array}{l}\text { Islam, Mohammad } \\
\text { Mizanul (TCPA) }\end{array}$ \\
\hline
\end{tabular}

Molina, Pablo (NENP)

Loaharanu, Paisan

(NAFA)

Gonfiantini, Roberto

(NAPC)

Lemmel, Hans Dietrich

(NAPC)

Muranaka, Richard

George G. (NAPC)

Piyasena, Rienzil

Dodwell (NAHU)

Ahmed, Jasim Uddin

(NENS)

Bates, Olan Gene

Gene (SGDE)

Wang, Dahai (NAPC)

Crijns, Martinus $\mathrm{J}$

(NEFW)

Alcala-Ruiz, Francisco

(NSNI)

Muranaka, Richard

George G. (NAPC)

Tauchid, Mohamad

(NEFW)

Markovic, Vitomir M. (NAPC)
1574988

134493

8016

162836

14811

218118

159157

87664

256731

75664

310762

112713 
INS1016 Radionuclide Standardization

INS1018 Neutron Activation Analysis

INS4017 Fuel Element Technology

INS4025

Quality Assurance and Quality Control Programmes

INS5020

Soil Water Management Studies

INS8015

Radiation Processing with an Electron Beam Machine

INS0010

Nuclear Information System

INS4026

Neutron Transmutation Doping of Silicon

INS6007 Quality Control and Maintenance of Nuclear Medical Equipment

INS9014

Research Reactor Safety

INS3009

Uranium Exploration and Development

INS8013

Radiocarbon Dating

INS0007

INS7002

Energy Economics Calculations with Nuclear Options Radiobiological Laboratory
1991-12-10 Islam, Mohammad Mizanul (TCPA)

\begin{tabular}{|c|c|}
\hline $991-12-10$ & $\begin{array}{l}\text { Islam, Mohammad } \\
\text { Mizanul (TCPA) }\end{array}$ \\
\hline $1991-12-17$ & $\begin{array}{l}\text { Islam, Mohammad } \\
\text { Mizanul (TCPA) }\end{array}$ \\
\hline $1991-12-17$ & $\begin{array}{l}\text { Islam, Mohammad } \\
\text { Mizanul (TCPA) }\end{array}$ \\
\hline $1991-12-17$ & $\begin{array}{l}\text { Islam, Mohammad } \\
\text { Mizanul (TCPA) }\end{array}$ \\
\hline $1991-12-17$ & $\begin{array}{l}\text { Islam, Mohammad } \\
\text { Mizanul (TCPA) }\end{array}$ \\
\hline 1991-12-17 & $\begin{array}{l}\text { Islam, Mohammad } \\
\text { Mizanul (TCPA) }\end{array}$ \\
\hline $1991-12-31$ & $\begin{array}{l}\text { Islam, Mohammad } \\
\text { Mizanul (TCPA) }\end{array}$ \\
\hline $1992-12-30$ & $\begin{array}{l}\text { Islam, Mohammad } \\
\text { Mizanul (TCPA) }\end{array}$ \\
\hline $992-12-30$ & $\begin{array}{l}\text { Islam, Mohammad } \\
\text { Mizanul (TCPA) }\end{array}$ \\
\hline $1992-12-30$ & $\begin{array}{l}\text { Islam, Mohammad } \\
\text { Mizanul (TCPA) }\end{array}$ \\
\hline $1992-12-30$ & $\begin{array}{l}\text { Islam, Mohammad } \\
\text { Mizanul (TCPA) }\end{array}$ \\
\hline 1994-04-18 & Sun, Yizhen (TCPA \\
\hline 1994-04-18 & Sun, Yizhen (TCPA) \\
\hline $1994-05-25$ & Sun, Yizhen (TCPA) \\
\hline 1994-05-25 & Sun, Yizhen (TCF \\
\hline
\end{tabular}

Squires, David J.

(NENF)

Ahmed, Jasim Uddin

(NENS)

Squires, David J.

(NENF)

Haider, Johann Georg

(TCPM)

Mani, Raghunath

Subra (NAPC)

Chantoin, Pierre $\mathrm{M}$.

(NEPF)

Pieroni, Nestor

Baltasar (NENP)

Kumarasinghe,

Kumarasinghege

Saliya (NAFA)

Markovic, Vitomir M.

(NAPC)

Taoka, Wesley M

(NESI)

Akhtar, Khalid

Mahmud (NAPC)

Xie, Yanfeng (NAHU)

55628

114005

150579

151989

68728

459344

31743

68993

87417

Alcala-Ruiz, Francisco

(NSNI)

Tauchid, Mohamad

(NEFW)

Rozanski, Kazimierz (NAML)

Molina, Pablo (NENP)

Mukherjee, Ramendra

Nath (NAHU)

11010

28
39133

- 71




\begin{tabular}{|c|c|c|c|c|}
\hline INS9016 & Environmental Radioactivity Measurements (Phase II) & $9 G$ & $1994-05-25$ & Sun, Yizhen (TCPA) \\
\hline INS9017 & Establishment of a Decontamination Facility & $9 G$ & $1994-05-25$ & Sun, Yizhen (TCPA) \\
\hline INS1015 & Utilization of Multi-Purpose Research Reactor & $\begin{array}{l}1 \mathrm{~L} \\
0 \mathrm{H}\end{array}$ & $1994-10-27$ & Sun, Yizhen (TCPA) \\
\hline INS4023 & Research Reactor Calculations and Experiments & $\begin{array}{l}4 A \\
4 C \\
4 D \\
4 B\end{array}$ & $1994-10-28$ & Sun, Yizhen (TCPA) \\
\hline INS4024 & Cyclotron Production of Radionuclides & $4 \mathrm{H}$ & 1994-11-07 & Sun, Yizhen (TCPA) \\
\hline INS6006 & Radiation Therapy & $6 C$ & 1994-11-07 & Sun, Yizhen (TCPA) \\
\hline INS5021 & Agricultural Production, Phase II & $5 \mathrm{~A}$ & $1995-04-24$ & Aslam, Javed (TCPA) \\
\hline INS6009 & Establishment of a Radioimmunoassay Laboratory & $6 \mathrm{~B}$ & $1995-05-15$ & Sun, Yizhen (TCPA) \\
\hline INS6008 & Medical Physics & $6 \mathrm{~F}$ & $1995-05-17$ & Sun, Yizhen (TCPA) \\
\hline INS9015 & Regulatory Aspect of Radiation Protection & $9 C$ & $1995-05-25$ & Sun, Yizhen (TCPA) \\
\hline INS4022 & Experimental Silicide Fuel Element & $4 \mathrm{E}$ & $1995-05-30$ & Sun, Yizhen (TCPA) \\
\hline INS6004 & Nuclear Medicine Services & $6 \mathrm{~B}$ & $1995-06-30$ & Sun, Yizhen (TCPA) \\
\hline INS0009 & Training in Nuclear Science and Technology & $\begin{array}{c}01 \\
1 \mathrm{~A} \\
4 \mathrm{~A}\end{array}$ & $1995-12-22$ & Sun, Yizhen (TCPA) \\
\hline
\end{tabular}
Fujimoto, Kenzo
(NENS)
Stegnar, Peter
(NSRW)
Laraia, Michele
(NEFW)
Akhtar, Khalid
Mahmud (NAPC)
Akhtar, Khalid
Mahmud (NAPC)

Vera Ruiz, Hernan
Guido (NAPC)

Llamas Mendoza,

Augusto Jose (NAHU)

García Podesta, Mario

(NAAL)

Kumarasinghe,

Kumarasinghege

Saliya (NAFA)

Piyasena, Rienzil

Dodwell (NAHU)

Llamas Mendoza,

Augusto Jose (NAHU)

Ortiz Lopez, Pedro

(NSRW)

Griffith, Richard V.

(NSRW)

Stegnar, Peter

(NSRW)

Akhtar, Khalid

Mahmud (NAPC)

Gopinathan Nair,

Gopalan (NAHU)

Walsh, Robin L.

(NAPC)
83663

37906

436988

235137

146408

189007

446461

68276

87814

53655

115001

119204

349458 


\begin{tabular}{|c|c|c|c|c|}
\hline INS8017 & Radiation Processing of Medical Products & $8 \mathrm{H}$ & $1995-12-22$ & Sun, Yizhen (TCPA) \\
\hline INS8019 & Isotopic Ratios in Geothermal Exploration & $8 \mathrm{M}$ & $1995-12-22$ & Sun, Yizhen (TCPA) \\
\hline INS9013 & Strengthening Nuclear Safety Infrastructure & $9 \mathrm{H}$ & $1995-12-22$ & Sun, Yizhen (TCPA) \\
\hline INS8018 & Radiation Processing Technology & $8 \mathrm{H}$ & 1996-02-29 & Sun, Yizhen (TCPA) \\
\hline INS9019 & Radiomedical Dosimetry & $\begin{array}{l}6 \mathrm{~F} \\
9 \mathrm{C}\end{array}$ & $1996-07-26$ & Sun, Yizhen (TCPA) \\
\hline INS1020 & Neutron Scattering Data Processing & $1 \mathrm{E}$ & $1996-09-25$ & Sun, Yizhen (TCPA) \\
\hline INS2011 & Production of Labelled Isotopes & $2 \mathrm{~F}$ & $1996-09-25$ & Sun, Yizhen (TCPA) \\
\hline INS4029 & $\begin{array}{l}\text { Upgrading Safety Instrumentation of Bandung } \\
\text { Research Reactor }\end{array}$ & $\begin{array}{l}4 G \\
4 B \\
4 D\end{array}$ & $1996-09-25$ & Sun, Yizhen (TCPA) \\
\hline INS9018 & Internal Dosimetry Service & $\begin{array}{l}6 \mathrm{~F} \\
9 \mathrm{C}\end{array}$ & 1996-09-30 & Sun, Yizhen (TCPA) \\
\hline INS0012 & $\begin{array}{l}\text { Energy Economic Calculations with Nuclear Option } \\
\text { (Phase II) }\end{array}$ & $\mathrm{OE}$ & $1996-10-29$ & $\begin{array}{l}\text { Kita, Tomohiko } \\
\text { (TCPA) }\end{array}$ \\
\hline INS0013 & Manpower Development & $O A$ & $1996-10-29$ & $\begin{array}{l}\text { Kita, Tomohiko } \\
\text { (TCPA) }\end{array}$ \\
\hline INS1021 & Utilization of Cyclotron & $1 \mathrm{~L}$ & $1996-10-29$ & $\begin{array}{l}\text { Kita, Tomohiko } \\
\text { (TCPA) }\end{array}$ \\
\hline INS4030 & $\begin{array}{l}\text { Research Reactor Calculations and Experiments - } \\
\text { Phase II }\end{array}$ & $\begin{array}{l}4 \mathrm{~A} \\
4 \mathrm{D} \\
4 \mathrm{~B}\end{array}$ & $1996-10-29$ & $\begin{array}{l}\text { Kita, Tomohiko } \\
\text { (TCPA) }\end{array}$ \\
\hline INS2013 & Instrumental Chemical Analysis & $2 \mathrm{C}$ & $1997-02-28$ & $\begin{array}{l}\text { Kita, Tomohiko } \\
\text { (TCPA) }\end{array}$ \\
\hline INS4027 & Reactor Physics Codes & $4 \mathrm{~B}$ & $1997-09-27$ & $\begin{array}{l}\text { Kita, Tomohiko } \\
\text { (TCPA) }\end{array}$ \\
\hline
\end{tabular}

Markovic, Vitomir M. (NAPC)

Gerardo-Abaya, Jane (TCLA)

Alcala-Ruiz, Francisco (NSNI)

Mrabit, Khammar (NSRW)

Markovic, Vitomir M. (NAPC)

Turai, Istvan (NSRW) Ortiz Lopez, Pedro (NSRW)

Dolan, Thomas

(NSRW)

Walsh, Michael A.R. (NAPC)

Dolan, Thomas (NSRW)

Gustafsson, Monica (NSRW) Griffith, Richard V. (NSRW)

Molina, Pablo (NENP)

Vera Ruiz, Hernan

Guido (NAPC)

Dolan, Thomas

(NSRW)

Walsh, Michael A.R

(NAPC)

Dolan, Thomas

(NSRW)
175542

280597

210852

226056

86900

153992

103374

29565

135024

77834

127320

56278

119985

191518

87034 


\begin{tabular}{|c|c|c|c|c|c|c|}
\hline INS9012 & Nuclear Power Plant Siting & $9 \mathrm{~F}$ & $1997-12-23$ & $\begin{array}{l}\text { Kita, Tomohiko } \\
\text { (TCPA) }\end{array}$ & & $\begin{array}{l}\text { Guerpinar, Aybars } \\
\text { (NSNI) } \\
\text { Lederman, Luis (NS) }\end{array}$ \\
\hline INS5025 & Feasibility Study on Food Irradiation & $5 \mathrm{H}$ & $1998-08-28$ & $\begin{array}{l}\text { Kita, Tomohiko } \\
\text { (TCPA) }\end{array}$ & & $\begin{array}{l}\text { Loaharanu, Paisan } \\
\text { (NAFA) }\end{array}$ \\
\hline INS0011 & University Research and Teaching & $\begin{array}{l}01 \\
5 A \\
4 A\end{array}$ & $1998-11-12$ & $\begin{array}{l}\text { Kita, Tomohiko } \\
\text { (TCPA) }\end{array}$ & & $\begin{array}{l}\text { Salema, Manase Peter } \\
\text { (TCAP) }\end{array}$ \\
\hline INS5024 & Effective Use of Fertilizers in Rice Cultivation & $5 \mathrm{~B}$ & $1998-12-11$ & $\begin{array}{l}\text { Kita, Tomohiko } \\
\text { (TCPA) }\end{array}$ & & Serraj, Rachid (NAFA) \\
\hline INS5022 & University Research and Teaching in Agriculture & $5 \mathrm{~A}$ & 1999-09-14 & $\begin{array}{l}\text { Kita, Tomohiko } \\
\text { (TCPA) }\end{array}$ & & $\begin{array}{l}\text { Salema, Manase Peter } \\
\text { (TCAP) }\end{array}$ \\
\hline INS5023 & $\begin{array}{l}\text { Feed Supplementation for Increasing Livestock } \\
\text { Production }\end{array}$ & $5 \mathrm{E}$ & $1999-11-30$ & $\begin{array}{l}\text { Kita, Tomohiko } \\
\text { (TCPA) }\end{array}$ & & $\begin{array}{l}\text { Perera, Buthgamu } \\
\text { Mudiyanselage } \\
\text { Amithral Oswin } \\
\text { (NAFA) }\end{array}$ \\
\hline INS7003 & Radiation Sterilization of Human Tissue Grafts & $7 \mathrm{E}$ & $2000-08-30$ & Sun, Yizhen (TCPA) & Sun, Yizhen (TCPA) & $\begin{array}{l}\text { Tatsuzaki, Hideo } \\
\text { (NAHU) }\end{array}$ \\
\hline INS9020 & Environmental Monitoring System Development & $9 \mathrm{~K}$ & $2000-08-30$ & Sun, Yizhen (TCPA) & Sun, Yizhen (TCPA) & $\begin{array}{l}\text { Winkler, Guenther } \\
\text { Leopold (NS) }\end{array}$ \\
\hline INS0014 & $\begin{array}{l}\text { Manpower Development and Nuclear Technology } \\
\text { Support }\end{array}$ & 이 & 2000-10-31 & Sun, Yizhen (TCPA) & Sun, Yizhen (TCPA) & $\begin{array}{l}\text { Khan, Asghar Ali } \\
\text { (NAAL) }\end{array}$ \\
\hline INS6010 & Maintenance of Co-60 Radiation Therapy Facilities & $6 \mathrm{~A}$ & 2000-10-31 & Sun, Yizhen (TCPA) & Sun, Yizhen (TCPA) & $\begin{array}{l}\text { Pernicka, Frantisek } \\
\text { (NAHU) } \\
\text { Ortiz Lopez, Pedro } \\
\text { (NSRW) }\end{array}$ \\
\hline INS5026 & Mutation Breeding of Bananas & $5 \mathrm{C}$ & 2002-05-16 & $\begin{array}{l}\text { Sukasam, Kesrat } \\
\text { (TCAP) }\end{array}$ & $\begin{array}{l}\text { Sukasam, Kesrat } \\
\text { (TCAP) }\end{array}$ & $\begin{array}{l}\text { Nichterlein, Karin } \\
\text { (NAFA) }\end{array}$ \\
\hline INS4028 & Support for the First Nuclear Power Plant & $4 \mathrm{C}$ & 2002-08-02 & $\begin{array}{l}\text { Sukasam, Kesrat } \\
\text { (TCAP) }\end{array}$ & $\begin{array}{l}\text { Sukasam, Kesrat } \\
\text { (TCAP) }\end{array}$ & $\begin{array}{l}\text { Mazour, Thomas } \\
\text { Joseph (NENP) }\end{array}$ \\
\hline INS4031 & Radiation Waste Management Programme & 40 & 2002-08-02 & $\begin{array}{l}\text { Sukasam, Kesrat } \\
\text { (TCAP) }\end{array}$ & $\begin{array}{l}\text { Sukasam, Kesrat } \\
\text { (TCAP) }\end{array}$ & Burcl, Rudolf (NEFW) \\
\hline INS9021 & NPP Site Confirmation and Structural Safety & $9 \mathrm{~F}$ & 2002-08-02 & $\begin{array}{l}\text { Sukasam, Kesrat } \\
\text { (TCAP) }\end{array}$ & $\begin{array}{l}\text { Sukasam, Kesrat } \\
\text { (TCAP) }\end{array}$ & $\begin{array}{l}\text { Guerpinar, Aybars } \\
\text { (NSNI) } \\
\text { Contri, Paolo (NSNI) }\end{array}$ \\
\hline INS3011 & Uranium Exploration and Development Study & $3 \mathrm{E}$ & 2004-01-28 & $\begin{array}{l}\text { Sukasam, Kesrat } \\
\text { (TCAP) }\end{array}$ & $\begin{array}{l}\text { Sukasam, Kesrat } \\
\text { (TCAP) }\end{array}$ & $\begin{array}{l}\text { Erdi-Krausz, Gabor } \\
\text { (NEFW) }\end{array}$ \\
\hline
\end{tabular}

727973

48245

383715

241703

380249

144756

255391

199044

601383

59856

110747

503767

60608

168633

511125 


\begin{tabular}{|c|c|}
\hline INS5028 & $\begin{array}{l}\text { Biofertilizers to Increase Food Crop Yields from Acid } \\
\text { Soil }\end{array}$ \\
\hline INS5027 & Mutation Breeding of Ornamental Plants \\
\hline INS2014 & Production of Advanced Radiopharmaceuticals \\
\hline INS0016 & $\begin{array}{l}\text { Comparative Assessment of Different Energy Sources } \\
\text { for Electricity Generation }\end{array}$ \\
\hline INS0015 & $\begin{array}{l}\text { Human Resource Development and Nuclear } \\
\text { Technology Support }\end{array}$ \\
\hline INS8021 & $\begin{array}{l}\text { Pilot-Scale Demonstration of Biomaterial for Medical } \\
\text { Care }\end{array}$ \\
\hline INS5029 & $\begin{array}{l}\text { Supplementary Feeding and Reproduction } \\
\text { Management of Cattle }\end{array}$ \\
\hline
\end{tabular}

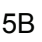

$5 C$

\section{$5 \mathrm{C}$}

\section{4-01-28}

2004-03-3

2004-04-30

2004-12-22

2005-01-3

2005-01-3

2005-02-28
Sukas (TCAP)

Sukasam, Kesrat (TCAP)

Sukasam, Kesrat (TCAP)

Sukasam, Kesrat (TCAP) (TCAP)

Sukasam, Kesrat (TCAP)

Sukasam, Kesrat (TCAP)
Sukasam, Kesrat
Sukasam, Kesrat (TCAP)

Sukasam, Kesrat (TCAP)

Sukasam, Kesrat (TCAP)

Rogner, Hans-Holge (NE)

Sukasam, Kesra (TCAP)

Sukasam, Kesra (TCAP)

Sukasam, Kesrat (TCAP)
Moutonnet, Pierre (NAFA)

Jain, Shri Mohan

(NAFA)

Pillai, Maroor

Raghavan

Ambikalmajan (NAPC)

Rogner, Hans-Holger

(NE)

Dias, Mahendra

Chmielewski, Andrzej

Grzegorz (NAPC)

Perera, Buthgamu

Mudiyanselage

Amithral Oswin

(NAFA)

Makkar, Harinder Paul

Singh (NAFA)
Prinath (TCAP)

\section{Cancelled National Projects (9)}

$\begin{array}{ll}\begin{array}{l}\text { Project } \\ \text { Number }\end{array} & \text { Title } \\ \text { INS5019 } & \text { Neutron Irradiation Facility }\end{array}$

INS9011

National Marine Radioactivity Research

INS1019

INS1022

INS2012

\section{Standardization of Radionuclides}

Design and Development of Neutron Generator Radionuclides in Food and Environmental Samples

\section{Fie}

\section{$5 \mathrm{C}$}

${ }_{1 \mathrm{C}}^{5}$

$9 \mathrm{E}$

$1 \mathrm{~K}$

$1 \mathrm{~L}$

9K 2C

\section{On}

\section{Cancelled}

$1991-10-15$

Mizanul (TCPA)

1993-10-12 Sun, Yizhen (TCPA)

1993-10-12 Sun, Yizhen (TCPA)

1993-10-12
Sun, Yizhen (TCPA)
70764

51492

217154

144778

525724

75216

271010

\section{Project Responsible Technical Officer}

\section{Officer}

Brunner, Helmut

(NAAL)

Micke, Alexander ()

Mrabit, Khammar (NSRW)

Haider, Johann Georg (TCPM)

Wang, Dahai (NAPC)

Mani, Raghunath

Subra (NAPC)

\section{Total Budget}




$\begin{array}{ll}\text { INS3010 } & \text { Uranium Exploration and Development in Kalan } \\ \text { INS8020 } & \text { Isotope Fractionation for Radiocarbon Dating } \\ \text { INS4032 } & \begin{array}{l}\text { Operation and Application of Low Energy } \\ \text { Accelerators }\end{array} \\ \text { INS8022 } & \begin{array}{l}\text { Radiotracers and Sealed Sources for Industry } \\ \text { Application }\end{array}\end{array}$

\subsection{Regional/Interregional Projects:}

$\begin{array}{ll}\begin{array}{c}\text { Active Regional/Interregional Projects (56) } \\ \text { Project } \\ \text { Number } \\ \text { INT0060 }\end{array} & \begin{array}{l}\text { Technical Co-operation Between Developing } \\ \text { Countries }\end{array} \\ \text { INT4131 } & \begin{array}{l}\text { Sustainable Technologies for Managing Radioactive } \\ \text { Wastes }\end{array} \\ \text { RAS6029 } & \begin{array}{l}\text { Distance-assisted Training for Nuclear Medicine } \\ \text { Technicians (RCA) }\end{array} \\ \text { INT5145 } & \begin{array}{l}\text { Insect Pest Control Using the Sterile Insect } \\ \text { Technique }\end{array} \\ \text { RAS5035 } & \begin{array}{l}\text { Improving Animal Productivity and Reproductive } \\ \text { Efficiency (RCA) }\end{array} \\ & \begin{array}{l}\text { Distance Education in Radiation Oncology (RCA) } \\ \text { RAS6033 }\end{array} \\ \text { RAS8084 } & \begin{array}{l}\text { Isotope Use in Managing and Protecting Drinking } \\ \text { Water (RCA) }\end{array}\end{array}$

$\begin{array}{crl}\text { 3C } & 1993-10-12 & \text { Sun, Yizhen (TCPA) } \\ \text { 3B } & & \\ \text { 3F } & & \\ 80 & 1993-10-12 & \text { Sun, Yizhen (TCPA) } \\ \text { 4G } & 2000-12-13 & \text { Sun, Yizhen (TCPA) } \\ \text { 8J } & 2003-11-01 & \begin{array}{l}\text { Sukasam, Kesrat } \\ \text { (TCAP) }\end{array}\end{array}$

1st Year of Approval

\section{Salema, Manase Peter (TCAP) \\ 1997 Kurghinyan, Vladimir (TCEU)}
1997 Dias, Mahendra Prinath (TCAP)
1999 Salema, Manase Peter (TCAP)
1999 Dias, Mahendra Prinath (TCAP)
1999 Dias, Mahendra Prinath (TCAP)
1999 Dias, Mahendra Prinath (TCAP)

Tauchid, Mohamad (NEFW)

Rozanski, Kazimierz (NAML)

Fazinic, Stjepko

(NAPC)

Sukasam, Kesrat

Jin, Joon-Ha (NAPC)
0

0

\begin{tabular}{|c|c|c|}
\hline \multirow{2}{*}{$\begin{array}{l}\text { Project Responsible } \\
\text { Officer }\end{array}$} & Technical Officer & Total Budget \\
\hline & $\begin{array}{l}\text { Salema, Manase Peter } \\
\text { (TCAP) }\end{array}$ & 946144 \\
\hline $\begin{array}{l}\text { Kurghinyan, Vladimir } \\
\text { (TCEU) }\end{array}$ & $\begin{array}{l}\text { Al-Mughrabi, } \\
\text { Mohamed (NEFW) } \\
\text { Balla, Janos (NEFW) } \\
\text { Kahraman, Alper } \\
\text { (NEFW) }\end{array}$ & 2565864 \\
\hline \multirow[t]{4}{*}{$\begin{array}{l}\text { Dias, Mahendra } \\
\text { Prinath (TCAP) }\end{array}$} & $\begin{array}{l}\text { Dondi, Maurizio } \\
\text { (NAHU) } \\
\text { Watanabe, Naoyuki } \\
\text { (NAHU) }\end{array}$ & 636688 \\
\hline & $\begin{array}{l}\text { Hendrichs, Jorge } \\
\text { Pedro (NAFA) }\end{array}$ & 1936300 \\
\hline & $\begin{array}{l}\text { Boettcher, Paul John } \\
\text { (NAFA) } \\
\text { Vercoe, Philip Edward } \\
\text { (NAFA) }\end{array}$ & 1052981 \\
\hline & $\begin{array}{l}\text { Hendry, Jolyon } \\
\text { Hindson (NAHU) }\end{array}$ & 715200 \\
\hline $\begin{array}{l}\text { Dias, Mahendra } \\
\text { Prinath (TCAP) }\end{array}$ & $\begin{array}{l}\text { Kulkarni, Kshitij } \\
\text { Mahadev (NAPC) }\end{array}$ & 589625 \\
\hline
\end{tabular}


Pre-Project Assistance (Africa and East Asia and

INT0074 the Pacific)

INT0076

Country Programme Review (Africa and East Asia and the Pacific)

RAS0032 Support Towards Self-Reliance and Sustainability of National Nuclear Institutions

RAS5039

Restoration of Soil Fertility and Sustenance of Agricultural Productivity (RCA)

RAS5040

Enhancement of Genetic Diversity in Food, Pulses, and Oil Crops and Establishment of Mutant Germplasm Network (RCA)

RAS6034

Quality Assurance Programme for Molecular-Based Diagnosis of Infectious

RAS8091

RAS8092

Diagnostics and Optimization in

Petrochemical Industry (RCA)

RAS8093

Geotherma Environment

Use of Isotopes in Dam Safety and Dam

Sustainability (RCA)

RAS9026
2001 Salema, Manase Peter (TCAP)

2001 Salema, Manase Peter (TCAP)

2001 Lutfi, Nabil (TCAP)

2001 Dias, Mahendra

2001 Dias, Mahendra Prinath (TCAP) Kim, Myung Ro (TCAP)

2001 Kamel, Reyad Sherif (TCAP)

2001 Dias, Mahendra Prinath (TCAP)

2001 Dias, Mahendra Prinath (TCAP)

2001 Dias, Mahendra Prinath (TCAP)

2001 Dey, Sujit (TCAP) Prinath (TCAP)
National Regulatory Control and Occupationa

Radiation Protection Programmes utfi, Nabil (TCAP)

Vez Carmona, Maria de Lourdes (EXPO) Djermouni, Belkacem (NSRW)

Salema, Manase Peter Salema, Manase Peter (TCAP)

Salema, Manase Peter (TCAP)

Dias, Mahendra Prinath (TCAP) Lutfi, Nabil (TCAP)

Zapata, Felipe (NAFA)

Shu, Qingyao (NAFA) Lokko, Yvonne Rosaline Naa (NAFA)

Lagoda, Pierre Jean

Laurent (NAFA)
Dey, Sujit (TCAP)

Kamel, Reyad Sherif (TCAP)

Dias, Mahendra

Prinath (TCAP)

Dias, Mahendra

Prinath (TCAP)

Dias, Mahendra

Prinath (TCAP)
619203

327847

539136

109200

660166

607540

863110

723375

287826

307631

1719895
Khan, Baldip Kaur

(NAHU)

Jin, Joon-Ha (NAPC)

Einav, Isaac (NAPC)

Kulkarni, Kshitij

Mahadev (NAPC)

Araguas Araguas, Luis

Jesus (NAPC)

Kurttas, Tuerker

(NAPC)

Mrabit, Khammar

(NSRW)

O'Donnell Torroba,

Patricio Alfonso

(NSRW) 
INT4141 Applications of Innovative Reactor Concepts for Developing Countries

INT5148

Establishing Quality Systems in Veterinary Testing Laboratories

RAS0035

Management of Technical Cooperation among

Developing Countries (RCA)

RAS0036

Country and Regional Programme Review

RAS0038

RAS4022

Role of Nuclear Power and Other Energy Options in Competitive Electricity Markets (RCA)

RAS4023

prevement of Research Reactor Operation and Utilization, Phase II (RCA)

RAS6038

Strengthening Medical Physics through Education and Training (RCA)

RAS7011 Enhancing the Sustainability of the Marine Coastal Environment (RCA)

\section{Salema, Manase Pete (TCAP)} Alain Jorge (TCEU)

2003 Salema, Manase Peter (TCAP)

\section{Dias, Mahendra Prinath (TCAP) \\ 2003 Salema, Manase Peter} (TCAP)

2003 Dias, Mahendra Prinath (TCAP)

2003 Dias, Mahendra Prinath (TCAP)

2003 Lutfi, Nabil (TCAP)

2003 Dias, Mahendra Prinath (TCAP)

2003 Dias, Mahendra Prinath (TCAP)

2003 Dias, Mahendra Prinath (TCAP)
2003 Cardoso Cabezon,

\section{Dey, Sujit (TCAP)}

abezon, Alain Jorge (TCEU)

Dias, Mahendra Prinath (TCAP)

Salema, Manase Peter (TCAP)

Dias, Mahendra

Prinath (TCAP)

Dias, Mahendra

Prinath (TCAP)

Lutfi, Nabil (TCAP)

Dias, Mahendra

Prinath (TCAP)

Dias, Mahendra

Prinath (TCAP)

Dias, Mahendra

Prinath (TCAP)
Mrabit, Khammar

(NSRW)

O'Donnell Torroba

Patricio Alfonso

(NSRW)

Hendry, Jolyon

Hindson (NAHU)

Kuznetsov, Vladimir

(NENP)

Viljoen, Gerrit

Johannes (NAFA)

Crowther, John

Richard (NAFA)

Dias, Mahendra

Prinath (TCAP)

Salema, Manase Peter

(TCAP)

Jalal, Ahmed Irej (NE)

Paranjpe, Shriniwas

Krishnarao (NAPC)

Kaufmann, Herbert

(NAPC)

McLean, Ian Donald

(NAHU)

Boisson, Florence

(NAML)

Jeffree, Ross A

(NAML)

Trinidad, Trinidad

Palad (NAHU)

Markowicz, Andrzej

(NAAL)
2277735

844860

883991

229580

997867

481900

228735

209975

316670

477264

305888

810946 
RAS8095

RAS8096

RAS8097

RAS9028

RAS9029

RAS9031 Bulk Analysis Techniques (RCA) Assessment of Radiological Risks (RCA)

Harmonization of Radiation Protection, Phase IV (RCA)

ological Emergency Response (RCA) Related Techniques

RAS0041
Tracing Future Sustainable Paths through Nuclear and Other Energy Options (RCA)
2003 (TCAP)

$\begin{array}{cl}2003 & \begin{array}{l}\text { Dias, Mahendra } \\ \text { Prinath (TCAP) }\end{array} \\ 2003 & \begin{array}{l}\text { Dias, Mahendra } \\ \text { Prinath (TCAP) }\end{array} \\ 2003 & \begin{array}{l}\text { Dias, Mahendra } \\ \text { Prinath (TCAP) }\end{array} \\ 2003 & \begin{array}{l}\text { Dias, Mahendra } \\ \text { Prinath (TCAP) }\end{array} \\ 2003 & \text { Lutfi, Nabil (TCAP) } \\ 2003 & \begin{array}{l}\text { Dias, Mahendra } \\ \text { Prinath (TCAP) }\end{array}\end{array}$
Prinath (TCAP)

2004 Dias, Mahendra Prinath (TCAP)

2005 Boussaha, Ali (TCAF)
Sukasam, Kesrat (TCAP)

Dias, Mahendra Prinath (TCAP)

Dias, Mahendra Prinath (TCAP)

Dias, Mahendra Prinath (TCAP)

Dias, Mahendra Prinath (TCAP)

Lutfi, Nabil (TCAP)

Dias, Mahendra

Prinath (TCAP)

Dias, Mahendra Prinath (TCAP)

Boussaha, Ali (TCAF)

Lagoda, Pierre Jean

Laurent (NAFA)

Trinidad, Trinidad

Palad (NAHU)

Jin, Joon-Ha (NAPC)

Scholten, Jan

Christoph (NAML)

Sampa, Maria Helena de O. (NAPC)

Kulkarni, Kshitij Mahadev (NAPC)

Lederman, Luis (NS)

O'Donnell Torroba,

Patricio Alfonso

(NSRW)

Mrabit, Khammar (NSRW)

Telleria, Diego Miguel (NSRW)

Berkovskyy,

Volodymyr (NSRW)

O'Donnell Torroba,

Patricio Alfonso

(NSRW)

Dias, Mahendra

Prinath (TCAP)

Zombori, Peter (NS)

Mc Kenna, Thomas

(NSRW)

O'Donnell Torroba,

Patricio Alfonso

(NSRW)

Hendrichs, Jorge

Pedro (NAFA)

Dias, Mahendra

Jalal, Ahmed Irej (NE)
918284

327934

370400

125911

462337

219490

779884

224709 Prinath (TCAP) 
Sustainable Land Use and Management Strategies for Controlling Soil Erosion and Improving Soil and Water Quality (RCA)

RAS5044

Integrated Approach for Improving Livestock Production Using Indigenous Resources and Conserving the Environment (RCA)

RAS6040

Improvement in Quality of Radiotherapy for

Frequent Cancers in the Region (RCA)

RAS604

Preventing Osteoporosis and Promoting Bone Mass in Asian Populations Using a Food-based Approach (RCA)

RAS604

RAS6043

Tumour Imaging Using Radioisotopes (RCA)

Regional Screening Network for Neonata

Hypothyroidism, Phase II

RAS8098 Radiation Technology for Development of Advanced

Materials and for Protection of Health and the

Environment (RCA)

RAS8099

Radioisotope Technology for Natural Resource Exploration and Exploitation (RCA)

RAS8100

Advanced Industrial Radiography (RCA)

Developing Technical Capabilities for the Protection of Health and Safety of Workers Exposed to lonizing Radiation
2005 Dias, Mahendra Prinath (TCAP)

2005 Dias, Mahendra Prinath (TCAP) Kim, Myung Ro (TCAP)

2005 Dias, Mahendra Prinath (TCAP) Kim, Myung Ro (TCAP)

\section{5}

Dias, Mahendra Prinath (TCAP)

Dias, Mahendra Prinath (TCAP)

2005 Dias, Mahendra Prinath (TCAP) (TCAP)

2005 Dias, Mahendra Prinath (TCAP)

2005 Dias, Mahendra Prinath (TCAP)

2005 Dias, Mahendra Prinath (TCAP)
Dias, Mahendra Prinath (TCAP)

2005 Kamel, Reyad Sherif

2005 Dey, Sujit (TCAP)

Dias, Mahendra

Prinath (TCAP)

Dias, Mahendra

Prinath (TCAP)

Dias, Mahendra

Prinath (TCAP)

(TCAP)

Dias, Mahendra

Prinath (TCAP)

Dias, Mahendra

Prinath (TCAP)

Dias, Mahendra

Prinath (TCAP)
Paranjpe, Shriniwas

Krishnarao (NAPC)

Pillai, Maroor

Raghavan

Ambikalmajan (NAPC)

Fulajtar, Emil (NAFA)

Boettcher, Paul John

(NAFA)

Vercoe, Philip Edward

(NAFA)

Rosenblatt, Eduardo

(NAHU)

McLean, Ian Donald

(NAHU)

Kamel, Reyad Sherif

Dey, Sujit (TCAP)
227440

335763

441200

893346

398180

400020

409070

275560

323350

243530

197650
Davidsson, Lena

Margareta (NAHU)

Watanabe, Naoyuki

(NAHU)

Solanki, Kishor K.

(NAHU)

Sampa, Maria Helena

de O. (NAPC)

Jin, Joon-Ha (NAPC)

Einav, Isaac (NAPC)

Deboodt, Pascal P. A

(NSRW)

O'Donnell Torroba,

Patricio Alfonso

(NSRW)

(NSRW) 
RAS9034 Strengthening Radiological Protection of Patients and Medical Exposure Control

2005 Dey, Sujit (TCAP)

to a Radiological and Nuclear Emergency

9L

RAS9035

RAS9036

Strengthening National Regulatory Infrastructure for the Control of Radiation Sources

9T

$9 \mathrm{C}$

2005

Dey, Sujit (TCAP)

(n)

RAS9037

Education and Training in Support of Radiation

Protection Infrastructure

2005 Dey, Sujit (TCAP)

Dey, Sujit (TCAP)

$$
\begin{aligned}
& \text { Sadagopan, Geetha } \\
& \text { (NSRW) }
\end{aligned}
$$




\begin{tabular}{|c|c|c|c|c|}
\hline RAS5020 & $\begin{array}{l}\text { Food Irradiation Process Control and Acceptance } \\
\text { (RCA) }\end{array}$ & $5 \mathrm{H}$ & $1996-05-13$ & $\begin{array}{l}\text { Easey, John Frederick } \\
\text { (TCPM) }\end{array}$ \\
\hline RAS8071 & $\begin{array}{l}\text { Isotopes \& Rad. for Tech. \& Environ. Sustainable } \\
\text { Devp. (RCA) }\end{array}$ & $\begin{array}{l}8 \mathrm{H} \\
8 \mathrm{~J}\end{array}$ & $1998-04-17$ & $\begin{array}{l}\text { Aleta, Carlito Ronguillo } \\
\text { (TCPA) }\end{array}$ \\
\hline RAS7003 & Radiation Sterilization of Tissue Grafts (RCA) & $7 \mathrm{E}$ & $1998-08-28$ & $\begin{array}{l}\text { Aleta, Carlito Ronguillo } \\
\text { (TCPA) }\end{array}$ \\
\hline RAS4011 & Research Reactor Utilization (RCA) & 4B & $1998-08-31$ & $\begin{array}{l}\text { Aleta, Carlito Ronguillo } \\
\text { (TCPA) }\end{array}$ \\
\hline RAS6022 & $\begin{array}{l}\text { Strengthening Nuclear Medicine in RCA Member } \\
\text { States (RCA) }\end{array}$ & $6 \mathrm{~B}$ & $1998-08-31$ & $\begin{array}{l}\text { Aleta, Carlito Ronguillo } \\
\text { (TCPA) }\end{array}$ \\
\hline RAS8068 & $\begin{array}{l}\text { Isotopes and Radiation in Industry and Environment } \\
\text { (RCA) }\end{array}$ & $\begin{array}{l}8 \mathrm{C} \\
8 \mathrm{~J} \\
8 \mathrm{H} \\
8 \mathrm{E}\end{array}$ & $1998-08-31$ & $\begin{array}{l}\text { Aleta, Carlito Ronguillo } \\
\text { (TCPA) }\end{array}$ \\
\hline RAS8070 & $\begin{array}{l}\text { Isotopes and Radiation in Industry and the } \\
\text { Environment (RCA) }\end{array}$ & $\begin{array}{l}8 \mathrm{C} \\
8 \mathrm{H} \\
8 \mathrm{G}\end{array}$ & $1998-08-31$ & $\begin{array}{l}\text { Aleta, Carlito Ronguillo } \\
\text { (TCPA) }\end{array}$ \\
\hline RAS4008 & Nuclear Instrument Maintenance (RCA) & $4 G$ & $1998-09-30$ & $\begin{array}{l}\text { Aleta, Carlito Ronguillo } \\
\text { (TCPA) }\end{array}$ \\
\hline RAS6018 & Radioimmunoassay for Hepatitis B Diagnosis (RCA) & $6 \mathrm{~B}$ & $1998-12-23$ & $\begin{array}{l}\text { Aleta, Carlito Ronguillo } \\
\text { (TCPA) }\end{array}$ \\
\hline INT4133 & $\begin{array}{l}\text { Sharing Experience for Nuclear Power Util. in Dev. } \\
\text { Countries }\end{array}$ & $\begin{array}{l}4 \mathrm{~A} \\
4 \mathrm{~B}\end{array}$ & $1999-04-12$ & Samiei, Massoud (NA) \\
\hline RAS0021 & Nuclear Power Planning (RCA) & $\begin{array}{l}0 A \\
0 B \\
0 J\end{array}$ & $1999-11-30$ & $\begin{array}{l}\text { Aleta, Carlito Ronguillo } \\
\text { (TCPA) }\end{array}$ \\
\hline RAS8069 & $\begin{array}{l}\text { Isotopes and Radiation in Industry and the } \\
\text { Environment (RCA) }\end{array}$ & $\begin{array}{l}8 \mathrm{G} \\
8 \mathrm{~J} \\
8 \mathrm{H} \\
8 \mathrm{~N}\end{array}$ & $1999-11-30$ & $\begin{array}{l}\text { Aleta, Carlito Ronguillo } \\
\text { (TCPA) }\end{array}$ \\
\hline RAS0023 & $\begin{array}{l}\text { Energy, Electricity and Nuclear Power Planning } \\
\text { (RCA) }\end{array}$ & $\begin{array}{l}\mathrm{OA} \\
\mathrm{OB} \\
\mathrm{OJ}\end{array}$ & $1999-12-21$ & $\begin{array}{l}\text { Aleta, Carlito Ronguillo } \\
\text { (TCPA) }\end{array}$ \\
\hline
\end{tabular}

\begin{tabular}{|c|c|}
\hline $\begin{array}{l}\text { Loaharanu, Paisan } \\
\text { (NAFA) }\end{array}$ & 523847 \\
\hline $\begin{array}{l}\text { Thereska, Jovan } \\
\text { (NAPC) } \\
\text { Gueven, Olgun } \\
\text { (NAPC) } \\
\text { Walsh, Michael A.R. } \\
\text { (NAPC) }\end{array}$ & 2549371 \\
\hline $\begin{array}{l}\text { Tatsuzaki, Hideo } \\
\text { (NAHU) }\end{array}$ & 1050580 \\
\hline Dimic, Viktor (NAPC) & 368146 \\
\hline $\begin{array}{l}\text { Padhy, Ajit Kumar } \\
\text { (NAHU) }\end{array}$ & 326653 \\
\hline $\begin{array}{l}\text { Thereska, Jovan } \\
\text { (NAPC) }\end{array}$ & 780874 \\
\hline $\begin{array}{l}\text { Thereska, Jovan } \\
\text { (NAPC) }\end{array}$ & 1006110 \\
\hline Xie, Yanfeng (NAHU) & 1198709 \\
\hline $\begin{array}{l}\text { Ch'Ng, Soo Ling } \\
\text { (NAHU) }\end{array}$ & 922575 \\
\hline $\begin{array}{l}\text { Khan, Arshad } \\
\text { Muhammad (NE) } \\
\text { Koorapaty, Venkata } \\
\text { Mahadeva Rao (NE) }\end{array}$ & 49750 \\
\hline $\begin{array}{l}\text { Mazour, Thomas } \\
\text { Joseph (NENP) }\end{array}$ & 165258 \\
\hline $\begin{array}{l}\text { Thereska, Jovan } \\
\text { (NAPC) }\end{array}$ & 466434 \\
\hline $\begin{array}{l}\text { Mazour, Thomas } \\
\text { Joseph (NENP) } \\
\text { Hamilton, Bruce (NE) }\end{array}$ & 410427 \\
\hline
\end{tabular}


RAS9006

Strengthening of Radiation Protection Infrastructures (RCA)

RAS5030

Feed Supplementation and Animal Production Strategies

RAS0024

Project Formulation Meetings (RCA)

RAS5034

Irradiation As Sanitary \& Phytosanitary Food Treatment (RCA)

RAS8075 Geothermal Energy Resources and Environmental Management

RAS4019 Improving Research Reactor Operation and Utilization (RCA)

RAS8078 Nucleonic Control Systems and Tracers in Industry (RCA)

RAS8080 Better Mgt of Envir \& Industry Thru Isotope \& Rad Tech (RCA)

RAS0028

Comparative Assessmt of Electricity Generation Options (RCA)

RAS4016 Disposal of Radioactive Waste from Non-Power Sources (RCA)

RAS8089

Optimization of Mineral Resources Recovery Using Low Radioactivity Portable Nucleonic Gauges (RCA)

RAS0033

Role of Nuclear Power and Other Energy Options in Mitigating Greenhouse Gas Emissions (RCA)

RAS4020
$9 \mathrm{C}$

2000-11-29

Xu, Naicheng (TCAP)

Xu, Naicheng (TCAP)

2001-05-31

Aleta, Carlito Ronguillo TCPA)

$5 \mathrm{H}$

$8 \mathrm{~A}$

2003-01-30

$2003-01-30$

2003-01-30

$2003-02-26$

$2003-02-26$

Aleta, Carlito Ronguillo

(TCPA)

Oresegun, Modupe

Olusola (NSRW)

Mrabit, Khammar

(NSRW)

Perera, Buthgamu

Mudiyanselage

Amithral Oswin

(NAFA)

Aleta, Carlito Ronguillo

(TCPA)

$$
\text { (TCPA) }
$$

Aleta, Carlito Ronguillo

Aleta, Carlito Ronguillo

(TCPA)

Loaharanu, Paisan

(NAFA)

$\mathrm{Xu}$, Naicheng (TCAP)

$\mathrm{Xu}$, Naicheng (TCAP)

Pang, Zhonghe

(NAPC)

Aleta, Carlito Ronguillo Basu, Saibal (NAPC) (TCPA)

(TCPA)

Aleta, Carlito Ronguillo

(TCPA)

Thereska, Jovan

(NAPC)

(TCPA)

Aleta, Carlito Ronguillo

(TCPA)

Turner, Jeffrey

Vaughan Vaughan

(TCPA)

(NAPC)

Aleta, Carlito Ronguillo Kim, Kyoung-Pyo

(TCPA)

(TCPA)

Kim, Kyoung-Pyo

(TCPA)

Bui, Duy Thanh (NE)

Dayal, Ramesh

(NEFW)

Aleta, Carlito Ronguillo Aleta, Carlito Ronguillo

(TCPA)

(TCPA)

Thereska, Jovan

(NAPC)

Aleta, Carlito Ronguillo

(TCPA)

Kim, Kyoung-Pyo

(TCPA)

4B

$2003-02-26$ Utilization (RCA)

(TCPA)
Kim, Kyoung-Pyo

(TCPA)
Bui, Duy Thanh (NE)

Paranjpe, Shriniwas

Krishnarao (NAPC)
1597518

402890

384759

175852

237149

192778

391643

811067

436979

156601

312352

202399

238067 

Indust.(RCA)

RAS8087

Radiation Processing Application for Agrowaste

(RCA)

RAS7008

RAS5029 Graft (RCA)

Nuclear Techniques for the Promotion of Agroforestry Systems

RAS0030

Design and Formulation of Project Proposals

RAS7010 Measuring the Effectiveness of Multinutrient Supplementation

RAS0025 Technical Co-operation Among Developing Countries (RCA)

RAS5037

Mutational Enhancement for Genetic Diversity in Rice (RCA)

RAS0029

Radiation Protection and Networking (RCA)

RAS2009

Quality Assurance and Good Manufacturing Practice for Radiopharmaceuticals

RAS4017

Maintenance and Repair of Nuclear Instruments

Nuclear Medicine Applications (RCA)
$8 \mathrm{H}$

$7 E$
2003-02-26

2003-02-26

2003-04-29

2003-05-30

2003-06-25

2003-12-31

2004-04-30

2004-04-30

2004-05-20

2004-05-20

2004-05-20

2004-05-20
(TCPA)

(TCPA)

(TCPA) (TCPA)

Fowler, Scott W.

(NAML)

Boisson, Florence

(NAML)

Turner, Jeffrey

Vaughan Vaughan

(NAPC)

Thereska, Jovan

(NAPC)

Aleta, Carlito Ronguillo

(TCPA)

Chmielewski, Andrzej

Grzegorz (NAPC)

Aleta, Carlito Ronguillo

(TCPA)

Hendry, Jolyon

Hindson (NAHU)

Aleta, Carlito Ronguillo

(TCPA)

Keerthisinghe,

Deegala Gamini

(NAFA)

Razley, Mohd. Nordin

(TCPA)

lyengar, Govindaraja

Venkatesha (NAHU)

Dias, Mahendra

Prinath (TCAP)

Kim, Kyoung-Pyo

Prinath (TCAP)

Prinath (TCAP)

Lutfi, Nabil (TCAP)

Dias, Mahendra Prinath (TCAP)

(TCPA)

Prins, Mahendra

Lutfi, Nabil (TCAP)

Lutfi, Nabil (TCAP)

Shu, Qingyao (NAFA)

Sadagopan, Geetha (NSRW)

Pillai, Maroor

Raghavan

Ambikalmajan (NAPC)

Kaufmann, Herbert (NAPC)

Dias, Mahendra Prinath (TCAP)

Padhy, Ajit Kumar

(NAHU)
201587

532451

78907

920506

390965

667493

475399

490260

335110

176735

322754

596355

1932912

Solanki, Kishor K.

(NAHU) 
Dias, Mahendra Prinath (TCAP)

Yonezawa, Chushiro (NAHU)

Boisson, Florence

(NAML)

Markowicz, Andrzej

(NAAL)

Pang, Zhonghe

(NAPC)

Kemppainen, Markku

(TCPC)

Chmielewski, Andrzej

Grzegorz (NAPC)

Jin, Joon-Ha (NAPC)

RAS8082

sotopic and Related Techniques to Assess Air Pollution - Joint UNDP/RCA (RCA)

Dias, Mahendra

Prinath (TCAP)

Dias, Mahendra

Prinath (TCAP)

Markowicz, Andrzej

(NAAL)

Yonezawa, Chushiro

(NAHU)

RAS9018 Harmonization of Radiation Protection (RCA)

$9 \mathrm{C}$

2004-10-29

Dias, Mahendra

Prinath (TCAP)

Dias, Mahendra

Prinath (TCAP)

Djermouni, Belkacem

(NSRW)

Mrabit, Khammar

(NSRW)

2C 2005-02-28 Lutfi, Nabil (TCAP)

Lutfi, Nabil (TCAP)

Rossbach, Matthias $\mathrm{H}$ (NAPC)

INT4134 Integrated Nuclear Power and Desalination System Design

Kurghinyan, Vladimir (TCEU)

(NENP)

Methnani, Mabrouk

(NENP)

\section{$8 C \quad 2005-06-30 \quad$ Dias, Mahendra}

Prinath (TCAP)

$6 B$

$2005-12-28$

Dias, Mahendra Prinath (TCAP) 


\section{Cancelled Regional/Interregional Projects (1)}

Project

Title

Field

Cancelled

On

RAS0034

Graduate Training and Education in Nuclear Sciences
0

\section{Project Responsible \\ Officer}

Lutfi, Nabil (TCAP)
Technical Officer

Salema, Manase Peter (TCAP)

Lutfi, Nabil (TCAP)
Total Budget

\footnotetext{
1 “Progress in Technology Development for Conversion of Mo-99 Production-BATAN's (Indonesia) Conversion Program...,"May 2005, http://www.rertr.anl.gov/RERTR27/PDF/S8-5 Bakel.pdf.

2 "RPI-10, the Indonesian 10 MW Isotope Production Reactor," International Group on Research Reactors (IGORR) News, No. 9, June 1997, http://www.igorr.com/newsletters/igorr-nl09/igorr3.html

3 Jakarta Post, 28 June 2006.

4 http://www.usembassyjakarta.org/econ/ESTH highlight may june06.html

5 IAEA Technical Cooperation Projects Database (TC-PRIDE), Project Nos. INS/3/006, INS/3/009, INS/3/009, and INS/3/011

${ }^{6}$ IAEA World Distribution of Uranium Deposits Database (UDEPO)

${ }^{7}$ IAEA Nuclear Fuel Cycle Information System (http://www-nfcis.iaea.org); see also Kosim Affandi et al., "Tinjauan Proses Pengendapan Konsentrat Uranium Skala Pilot" ["Assessment of Uranium Concentrate Precipitation of on a Pilot Scale"], Prosiding Seminar Pranata Nuklir and Litkayasa, 2 September 1998 (Abstract in English available as abstract number 0910 on BATAN digital library Web site, http://digilib.batan.go.id/sipulitbang/sipulitbang.php)

${ }^{8}$ IAEA Technical Cooperation Projects Database (TC-PRIDE), Project No. INS/3/007.

${ }^{9}$ Indonesia Country Profile, Stockholm International Peace Research Institute (SIPRI) Counties of Strategic Nuclear Concern,

http://www.sipri.org/contents/expcon/cnsc3ins.html, citing the following reference which no longer is available at the indicated URL: http://www.grdc.esdm.go.id/Product/map/Kalimantan/Nangapinoh-des.asp .

${ }^{10}$ PTNBR Web site, http://www.batan-bdg.go.id

${ }^{11}$ IAEA Technical Cooperation Projects Database (TC-PRIDE), Project Nos. INS/4/015 and INS/4/017.

12 http://www.batan.go.id/ptapb/index-e.php

13 http://www.batan.go.id/en2006/facilities.php

${ }_{15}^{14}$ http://www.batan.go.id/patir

15 “Countries of Strategic Concern: Indonesia," SIPRI, July 2004, http://www.sipri.org/contents/expcon/cnsc3ins.html

16 "International GNEP Assessment: Indonesia," pp. 5-6.

17 "Nuclear Knowledge Management, Indonesia: National Nuclear Authorities," http://www.iaea.org/inis/ws/countries/indonesia.html

18 "International GNEP Assessment: Indonesia," p. 8.

${ }^{19}$ BATAN National Nuclear Agency, http://www.batan.go.id/batan/index_e.html

20 "Nuclear Authorities in Indonesia," http://www.iaea.org/inis/ws/nuclear_authorities/indonesia.html
} 


\footnotetext{
${ }^{21}$ Ministry of Research and Technology: Organization,

http://www.ristek.go.id/english/organization.html?bcsi scan 3ED561A05223E3E3=0\&bcsi scan filename=organization.html 22 “Asian Nuclear Safety Network," http://ansn.bapeten.go.id/

23 “IRTR Program Country Plan: Indonesia," FY06 Rev 0, 1 October 2005

${ }^{24}$ Ibid., pp.2-6.
} 